\title{
Quantifying Decoherence in Attosecond Metrology
}

\author{
C. Bourassin-Bouchet $\odot,{ }^{1, *}$ L. Barreau $\odot,{ }^{2}$ V. Gruson, ${ }^{2}$ J.-F. Hergott, ${ }^{2}$ F. Quéré $\odot,{ }^{2}$ P. Salières $\odot,{ }^{2}$ and T. Ruchon $\odot^{2}$ \\ ${ }^{1}$ Laboratoire Charles Fabry, Institut d'Optique Graduate School, CNRS, Université Paris-Saclay, \\ 91127 Palaiseau Cedex, France \\ ${ }^{2}$ LIDYL, CEA, CNRS, Université Paris-Saclay, CEA Saclay, 91191 Gif-Sur-Yvette, France
}

(Received 21 December 2018; revised 2 July 2020; accepted 8 July 2020; published 28 August 2020)

\begin{abstract}
Laser-dressed photoemission spectroscopy has established itself as the gold standard of attosecond temporal metrology. In this technique, the attosecond structure of an extreme-ultraviolet pulse is retrieved from the wave function of the electron wave packet released during photoionization. Here, we show that this electron wave packet should rather be described using the density matrix formalism, thus allowing one to account for all processes that can affect its coherence, from the attosecond pulse generation to the photoemission and the measurement processes. Using this approach, we reconstruct experimentally a partially coherent electron wave packet with a purity of 0.11 ( 1 for full coherence). Comparison with theoretical models then allows us to identify the origins of this decoherence and to overcome several limitations such as beam-line instabilities or spectrometer resolution. Furthermore, we show numerically how this method gives access to the coherence and eigencomponents of complex photoelectron wave packets. It thus goes beyond the current measurement of photoionization time delays and provides a general framework for the analysis and understanding of complex photoemission processes.
\end{abstract}

DOI: $10.1103 /$ PhysRevX.10.031048

Subject Areas: Atomic and Molecular Physics, Optics, Quantum Physics

\section{INTRODUCTION}

Physical objects with a wavelike behavior (a classical electric field, a quantum wave function, etc.) are often represented mathematically as the combination of an amplitude and a phase. From the observer's point of view, however, such a simple picture can sometimes fail to describe reality, e.g., when the contrast of an interference pattern remains inexplicably lower than expected. This apparent inconsistency finds a solution when introducing the notion of coherence, that is, when considering that the measurement has built itself through the accumulation of elementary waveforms with different amplitudes and phases. The statistical mixture composed of all these waveforms then becomes the relevant object of interest. In quantum mechanics, this change of paradigm is operated when replacing the wave function with the density matrix. The diagonal elements of this matrix, called the populations, give the probability of finding the particles in some state, whereas the off-diagonal elements, the coherences,

\footnotetext{
* Corresponding author. charles.bourassin-bouchet@institutoptique.fr

Published by the American Physical Society under the terms of the Creative Commons Attribution 4.0 International license. Further distribution of this work must maintain attribution to the author(s) and the published article's title, journal citation, and DOI.
}

provide the effectively observed interference terms between the states, thus describing the ensemble of wave packets in all its complexity.

This conceptual shift has not yet been introduced in attosecond metrology. The conventional scenario underlying present measurement techniques is fully coherent: in reconstruction of attosecond beatings by interference of two-photon transitions (RABBIT) [1], attosecond streaking $[2,3]$, frequency-resolved optical gating for complete reconstruction of attosecond bursts (FROG CRAB) [4-6], and phase retrieval by omega oscillation filtering (PROOF) [7], and, in most other techniques [8,9], the recovered information is the temporal (or, equivalently, spectral) amplitude and phase of an attosecond extreme-ultraviolet (XUV) waveform. Generally, the XUV pulse is first converted into an electron wave packet (EWP) via photoionization in the presence of a laser pulse with a controlled delay $[10,11]$. The attosecond electric field is then retrieved from the changes observed in the photoelectron kinetic energy spectrum when changing the laser delay. Beyond the characterization of ultrashort pulses, attosecond metrology has been used to probe photoionization dynamics in ever more complex systems in recent years [12-20], still in the fully coherent picture.

Nevertheless, from its birth to its detection, the EWP can accumulate the influence of many incoherent phenomena. Such decoherence can first arise due to a classical ensemble averaging. For example, the XUV pulse properties can vary during the measurement process due to space-time 
coupling or shot-to-shot fluctuations, photoelectrons can undergo inelastic scattering on neighboring particles during photoionization [21,22], the detector's response may alter the data by filtering out details in the spectrum, etc. In all these cases, the obtained photoelectron spectrum can be explained only if the EWP is described as a statistical ensemble associated to a density matrix.

Moreover, decoherence can also arise for quantummechanical reasons. If a physical phenomenon entangles the observed photoelectron with unobserved degrees of freedom of the system, the EWP appears in a mixed state. In this case, one can build only a reduced density matrix where, e.g., the degrees of freedom of the $N-1$ bound electrons and possibly molecular vibrational modes have been traced over [23]. Therefore, ionization processes involving electron-ion interactions [24], or electronelectron interactions (shake-up processes [19], autoionization [25], Auger processes [26], etc.), and more generally multichannel ionization processes [24,27-29], should be particularly prone to decoherence.

Interestingly, the question of coherence in attosecond ionization has been mainly discussed in the case where the observed subsystem was the remaining ion instead of the photoelectron. An early study investigated the coherence of a spin-orbit wave packet in an atomic ion produced through strong-field ionization [28]. More recently, the coherence of broad ionic wave packets produced in molecular ionization has been studied in the context of attosecond charge migration [16,30-34].

Here, we propose a complete reinterpretation of laserdressed photoionization to account for decoherence processes in attosecond metrology. To do so, we build upon a theoretical approach named Mixed FROG, initially designed to reconstruct statistical ensembles of classical ultrashort light pulses from FROG traces [35]. We show that, in the context of laser-dressed photoionization, Mixed FROG gives access to the full quantum state of the released EWP, represented by its density matrix or by its Wigner quasiprobability distribution. Reconstructing this quantum state makes it possible to decipher the quantum phenomena that unavoidably affect the EWP, such as state superpositions and decoherence, which have been overlooked until now in attosecond metrology. In many other domains, including atom optics [36], quantum electrodynamics [37], and quantum information [38,39], this approach is often called quantum state tomography. We present the first experimental demonstration of this technique in attophysics and compare the obtained density matrix with theoretical models in order to retrieve the origin of the observed decoherence. Finally, we illustrate on a numerical example how Mixed FROG could be used to probe the coherence in the case of multiple ionization channels. This work deepens our understanding of laser-dressed photoionization, opening new prospects for attosecond photoemission spectroscopy.

\section{RESULTS}

\section{A. Reconstructing mixtures of electron wave packets}

When the XUV pulse interacts with atoms from a gas jet, photoionization takes place; see Fig. 1(a). The emitted EWP can be described by a density matrix $\rho$ representing the ensemble of released single-electron wave packets. This quantum object inherits the statistical properties of the classical XUV electric field and can additionally be affected by decoherence effects from photoionization itself (unobserved collisions, multiple ionization channels, electronelectron correlations, etc.). Finally, after sufficient propagation, the single-electron wave packets spread in time, as their different kinetic energy components do not travel at the same velocity. Therefore, the time profile of the ensemble measured at a macroscopic distance from the source provides its energy spectrum, a technique called time-of-flight (TOF) spectroscopy. In this case, the limited TOF resolution of the spectrometer affects the measured signal. The final photoelectron spectrum denoted $\langle S\rangle$ is an average over the unobserved degrees of freedom at the macroscopic and atomic levels. We now explain how the density matrix $\rho$ can be obtained from measurements of $\langle S\rangle$.

In the scenario shown in Fig. 1, photoionization is triggered by an XUV attosecond pulse train (APT) corresponding to the superposition of three high-order harmonics, which thus populates three levels of the continuum equispaced in kinetic energy $\epsilon$ and denoted 1,3 , and 5 in Figs. 1(b) and 1(c). Measuring the photoelectron spectrum with a TOF detector gives the electron populations in each energy level, that is, the diagonal elements of the density matrix $\langle S(\epsilon)\rangle=\rho(\epsilon, \epsilon)$ (see the Appendix A). In order to get the off-diagonal elements, i.e., the coherences, one needs to interfere each populated state of the continuum with all the others. This interference is achieved by adding an external laser field with which the EWP interacts at the time of ionization. Considering either the particlelike or the wavelike behavior of the laser pulse leads to two equivalent interpretations of this interaction, described in Figs. 1(b) and 1(c).

Through the absorption or emission of several laser photons, electrons originally occupying two distinct levels with energies $\epsilon$ and $\epsilon^{\prime}$ can follow various quantum paths and end up on the same energy level. The resulting quantum interferences encode in the spectrum the amplitude and phase of the off-diagonal elements $\rho\left(\epsilon, \epsilon^{\prime}\right)$. More specifically, the case where the EWP emits or absorbs one laser photon only is the underlying process of the RABBIT technique used to measure the phase difference between two adjacent harmonics. In Mixed FROG, the key difference is that, by increasing gently the laser intensity, one induces multi-laser-photon transitions, which gives access to interference terms between nonconsecutive levels (higher-order coherences) [40]. When the intensity is such 


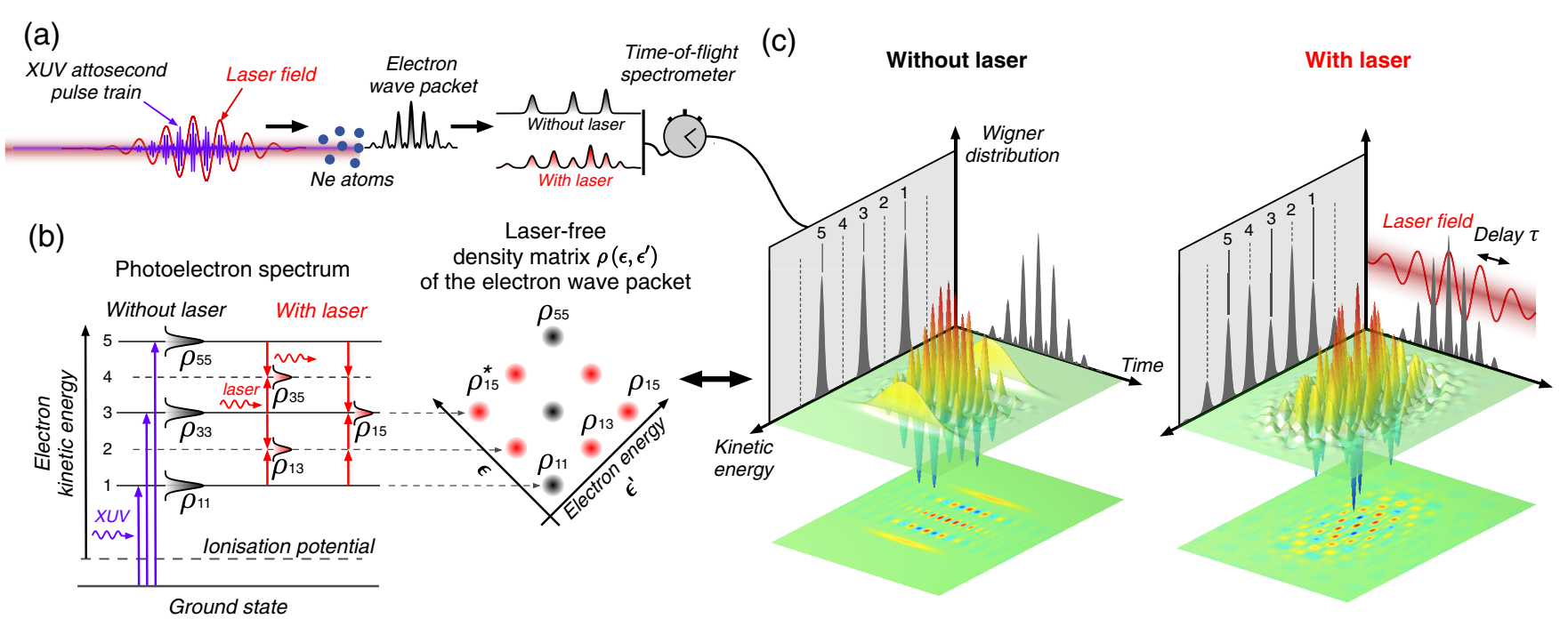

FIG. 1. Principle of a Mixed FROG measurement. (a) An XUV attosecond pulse train (purple curve) frees an electron wave packet (gray filled line) through photoionization. During this step, a laser pulse (red curve) modifies the wave packet, the kinetic energy spectrum of which is then measured with a time-of-flight spectrometer. (b) In the absence of a laser pulse, this spectrum gives the populations (the diagonal elements in black) of the laser-free density matrix $\rho$. Adding the laser pulse gives access to the off-diagonal elements of $\rho$ (in red) through the emission or absorption of laser photons. (c) The state of the photoelectron wave packet can equivalently be described by a Wigner distribution $W$ in the energy or time phase space. Measuring the energy spectrum for various XUV and laser delays provides a set of projections of $W$, which makes it possible to access the Wigner distribution by tomography.

that the populated states having the lowest and highest energy can interfere, there is fundamentally enough information at hand to reconstruct the full density matrix.

The same process can be pictured differently, this time representing the EWP with the help of a Wigner function $W(\epsilon, t)$, a quasiprobability distribution in the kinetic energy or time phase space which describes the mixture as completely as $\rho\left(\epsilon, \epsilon^{\prime}\right)$ (see Appendix A). In this framework, the photoelectron spectrum becomes the projection of $W$ along the $\epsilon$ axis, $\langle S(\epsilon)\rangle \propto \int W(\epsilon, t) d t$, whereas projecting $W$ on the time axis gives the EWP time profile in Fig. 1(c). In the standard interpretation of laser-dressed photoionization, the laser field induces a temporal phase modulation on the EWP, which modifies its kinetic energy distribution $[10,11]$. The evolution of the photoelectron spectrum with the laser or XUV delay $\tau$ is called a spectrogram. In the Wigner representation, the laser field modifies $W(\epsilon, t)$, which changes its projection $\langle S(\epsilon, \tau)\rangle$. Therefore, the photoelectron spectra composing the spectrogram can be rethought as a large ensemble of projections. If the laser field is intense enough, these projections encode the finest features of the Wigner function, enabling its full tomographic reconstruction from the spectrogram.

In practice, the Mixed FROG technique consists in processing a spectrogram recorded at sufficiently high laser intensity with an advanced mixture-retrieval algorithm [35]. As most techniques in attosecond metrology are already based on the acquisition of spectrograms, this approach implies minimal experimental modifications to reach the required laser intensity and then mostly relies on more advanced data processing.

\section{B. Experimental demonstration}

In order to validate these concepts, we implement Mixed FROG on the Plateforme Laser Femtoseconde Accordable attosecond beam line at CEA-LIDYL. The objective is to see if the EWP deviates from full coherence in routine experimental conditions [41], i.e., in the case of a single ionization channel, without active IR-XUV delay stabilization, and with standard TOF electron spectrometer detection. With this perspective in mind, an APT generated in neon is spectrally filtered with a double aluminum or zirconium foil to isolate a group of four odd harmonics (H39-H45) of the $800 \mathrm{~nm}$ driving laser. The APT is then focused into a neon gas jet where photoionization takes place. A dressing laser pulse (50 fs FWHM, $800 \mathrm{~nm}$ ) synchronized with the APT then modulates the electron energy spectrum, which is finally measured with a 2-m-long TOF electron spectrometer. A retarding potential of $24 \mathrm{~V}$ is applied to select the photoelectrons emitted from the $2 p$ neon valence shell. By observing the "outer" sidebands appearing in the photoelectron spectrum when increasing the dressing intensity, we could deduce the maximum number of laser photons involved in continuum-continuum transitions at this intensity; see Fig. 2(a). At $6 \mathrm{TW} \cdot \mathrm{cm}^{-2}$, this number of photons (approximately eight) is large enough to interfere the two outermost energy levels, a necessary condition to access the full density matrix. After measuring the spectrogram at this intensity, the inversion procedure yields the density matrix $\rho_{\exp }\left(\epsilon, \epsilon^{\prime}\right)$ and the Wigner distribution $W_{\exp }(\epsilon, t)$ in Figs. 2(b) and 2(c) (see Supplemental Material [42], parts I and II).

Looking at the projections of $W_{\text {exp }}$, one retrieves the four harmonics composing the photoelectron spectrum and the 


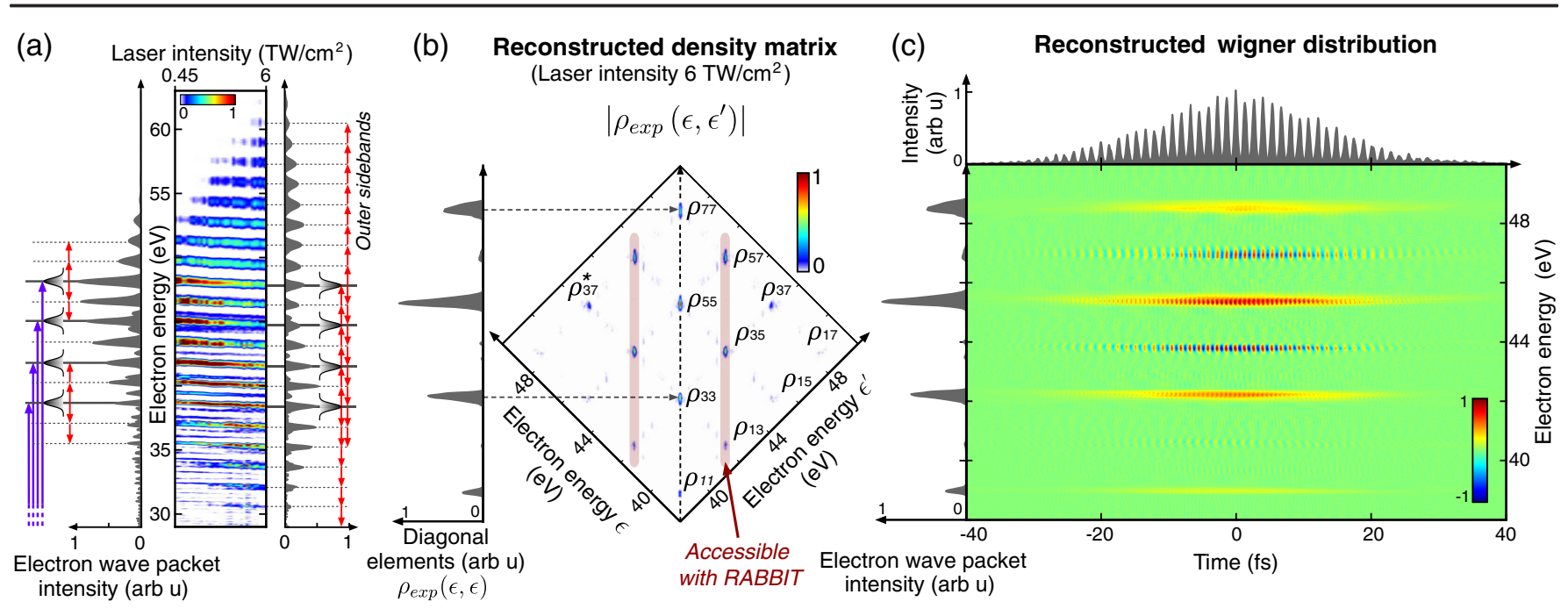

FIG. 2. Implementation of Mixed FROG for an attosecond pulse train generated in neon and photoionizing neon in the $2 p$ valence shell. (a) The left and right show the photoelectron spectra for laser intensities of $4.5 \times 10^{11}$ and $6 \times 10^{12} \mathrm{~W} \cdot \mathrm{cm}^{-2}$, respectively. The central panel gives the evolution of the spectrum between these two intensities: The linear drift in energy of the peaks observed when the intensity is increased corresponds to the laser-induced ponderomotive shift [43]. Increasing the dressing intensity enables the electron wave packet to emit or absorb more laser photons, which is confirmed by the appearance of numerous "outer" sidebands in the photoelectron spectrum [40,44]; see the right. When multi-laser-photon transitions allow the first and last populated levels to interfere, the highest-order coherence becomes encoded into the spectrum, and the full density matrix can be obtained. (b) Retrieved density matrix in the energy domain $\rho_{\text {exp }}\left(\epsilon, \epsilon^{\prime}\right)$ (only the modulus shown) for a dressing laser intensity of $6 \times 10^{12} \mathrm{~W} \cdot \mathrm{cm}^{-2}$. The dark gray shaded curve on the left gives the diagonal elements, that is, the laser-free photoelectron spectrum. (c) Reconstructed Wigner distribution $W_{\text {exp }}(\epsilon, t)$. Its projections onto the temporal (upper) and energy axes (left), respectively, correspond to the average time profile of the electron wave packet and the laser-free photoelectron spectrum.

temporal profile of the XUV APT that is imprinted onto the EWP. One of the most striking features of the experimental Wigner function is the presence of strong oscillations showing both positive and negative values. The presence of negative values in the Wigner function is usually considered as a sign of the quantum nature of the state $[37,45]$. In the present case, it shows that the EWP is indeed in a coherent superposition of four distinct energy states of the continuum. However, the density matrix elements $\rho_{\exp }\left(\epsilon, \epsilon^{\prime}\right)$ tend to zero as one moves away from the diagonal; see, for instance, $\rho_{15}$ and $\rho_{17}$. Such an offdiagonal-element disappearance is often referred to as decoherence and reveals the presence of a statistical ensemble of nonidentical single-electron wave packets. This result is confirmed by calculating the purity of the reconstructed mixture, $\iint\left|\rho_{\exp }\left(\epsilon, \epsilon^{\prime}\right)\right|^{2} d \epsilon d \epsilon^{\prime}=0.11$, instead of 1 for full coherence. We now explain the origin of this loss of coherence.

\section{The origin of decoherence}

For this analysis, it is more convenient to Fourier transform $\rho_{\exp }\left(\epsilon, \epsilon^{\prime}\right)$ and study the density matrix in the time domain $\rho_{\exp }\left(t, t^{\prime}\right)=\left\langle\chi(t) \chi^{*}\left(t^{\prime}\right)\right\rangle$ [46], where $\chi(t)$ corresponds to the time-dependent wave function of each single-electron wave packet composing the mixture; see Fig. 3(a). As in the energy domain, the diagonal elements of $\rho\left(t, t^{\prime}\right)$ give the average time profile of the wave packets. Off diagonal, the density matrix takes the form of a grid where each peak gives the interference term of the mixture at time $t$ with itself at another time $t^{\prime}$, i.e., the coherence between different attosecond pulses in the train. Strikingly, this grid vanishes when $\left|t-t^{\prime}\right| \gtrsim 10$ fs [see the red dashed lines in Fig. 3(a)], indicating that no interference can be observed if two events are separated in time by more than a few femtoseconds. The disappearance of the off-diagonal elements is also visible at the attosecond timescale. Indeed, the oval shape of the attosecond peaks indicates that the front of a peak cannot interfere with its tail; see the red dashed lines in the inset in Fig. 3(a).

In the conditions of the experiment, we estimate that such decoherence can result from a limited number of phenomena, namely, (i) fluctuations of the synchronization between the XUV and dressing pulses, e.g., due to beamline instabilities, (ii) variations of the XUV attosecond chirp which could originate from fluctuations of the APT generation conditions [47-49], and (iii) the limited timeof-flight resolution of the spectrometer [50]. In research fields where the density matrix and Wigner formalisms are used, it is common to compare experimental mixtures to analytical models in order to identify the physical origin of decoherence $[46,51,52]$ or simply to remove its influence from a state of interest [45]. We follow the same approach here and model analytically the phenomena mentioned 
(a)

Measured

(b)

Model fitted

\section{$\underset{\rho_{\text {exp }}(t, t)}{\text { Diagonal elements }}$}

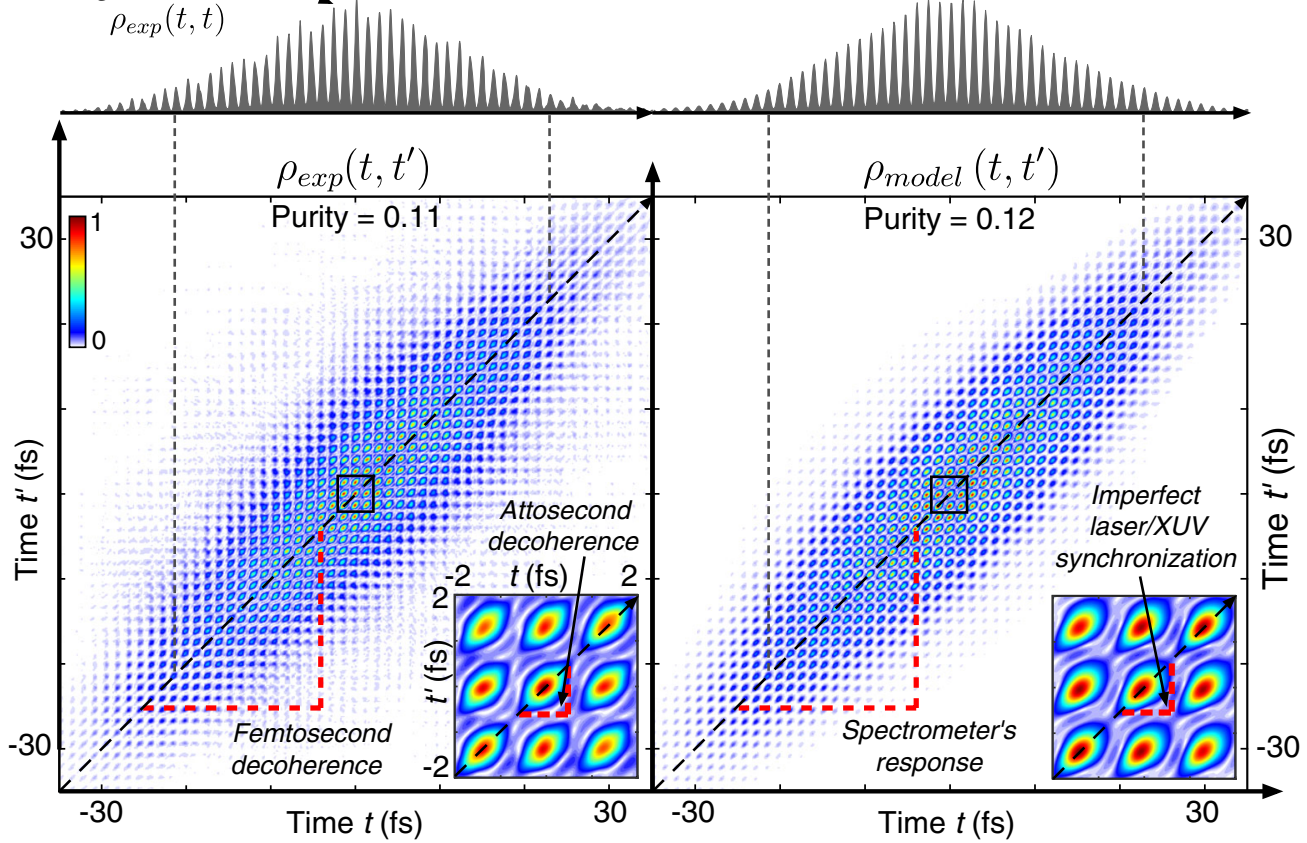

(c)

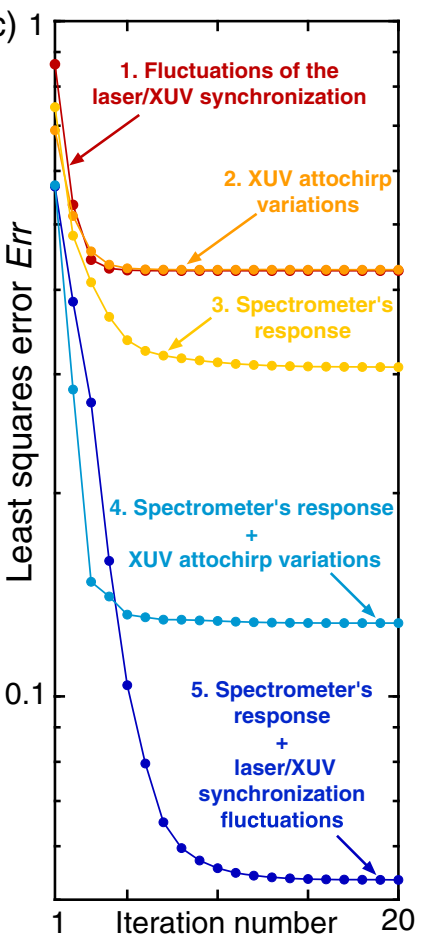

FIG. 3. Comparison to decoherence models. (a) Fourier transforming $\rho_{\exp }\left(\epsilon, \epsilon^{\prime}\right)$ provides the experimental density matrix in the time domain $\rho_{\text {exp }}\left(t, t^{\prime}\right)$; only the modulus is shown. Inset: Enlargement of the area delimited by the black square in the main panel. Its diagonal elements (upper) give the time structure of the electron wave packet. Off-diagonal-element disappearance, also called decoherence, can be observed at the femtosecond and attosecond timescales as emphasized by the red dashed lines. (b) To identify the physical origin of decoherence, several density matrix models $\rho_{\text {model }}$ are fitted onto $\rho_{\text {exp }}$. The best agreement (shown here) is obtained with model 5: The femtosecond decoherence comes from the limited spectrometer resolution, while the attosecond decoherence arises from fluctuations of the synchronization between the laser and XUV pulses in the spectrometer. (c) Evolution of the least-squares error $\iint\left|\rho_{\exp }-\rho_{\text {model }}\right|^{2} d t d t^{\prime}$ with the number of iterations of the fitting algorithm for various decoherence models.

above. In the fully coherent case, the ensemble contains identical single-electron wave packets $\chi_{0}(t)$ which factorize out of the averaging \langle\rangle in the density matrix expression, giving $\rho_{\text {model }}\left(t, t^{\prime}\right) \propto \chi_{0}(t) \chi_{0}^{*}\left(t^{\prime}\right)$. If the arrival time of the XUV pulse fluctuates with respect to the laser pulse, the mixture is composed of identical wave packets $\chi_{0}(t-T)$ that can be randomly delayed with a probability density $P(T)$, leading to a more complex density matrix model $\rho_{\text {model }}\left(\chi_{0}, P\right)$. Equivalently, models can be derived to account for variations of the XUV attosecond chirp and for the spectrometer response function, with respective probability densities denoted $C$ and $R$, giving rise to density matrices with the general form $\rho_{\text {model }}\left(\chi_{0}, P, C, R\right)$; see Appendix B. For each decoherence scenario, $\rho_{\text {model }}$ is fitted onto $\rho_{\exp }$ using an iterative algorithm detailed in Supplemental Material [42], part III. The problem being highly constrained, the algorithm rapidly converges toward the functions $\chi_{0}, P, C$, and $R$ that minimize Err $=\iint\left|\rho_{\text {exp }}-\rho_{\text {model }}\right|^{2} d t d t^{\prime}$. In the end, the decoherence model giving the lowest residual error Err is the one that best reproduces the observed mixture. The detailed results of this analysis are shown in Appendix C, and the leastsquares errors are reported in Fig. 3(c).
In models 1 and 2, decoherence is respectively assumed to arise from fluctuations of the synchronization between the laser and XUV pulses or from fluctuations of the XUV attosecond chirp. These phenomena affect the coherence at the attosecond timescale, i.e., the shape of the attosecond peaks in $\rho_{\exp }\left(t, t^{\prime}\right)$, but cannot explain the observed femtosecond decoherence, so that the fitting process leads to rather large residual errors. Model 3 considers the spectrometer's resolution as the sole source of decoherence. Doing so, one immediately reproduces the femtosecond decoherence, which reduces the error. Indeed, in this model, the limited resolution prevents one from resolving the spectral interferences that would originate from two ultrashort events with a time separation $\left|t-t^{\prime}\right|$ larger than approximately $10 \mathrm{fs}$. This result gives a simple physical explanation to the disappearance of high-order coherences in $\rho_{\text {exp }}\left(t, t^{\prime}\right)$. Finally, models 4 and 5 combine several of the previous sources of decoherence. The best fit is obtained when both the spectrometer's response and fluctuations of the synchronization between the laser and XUV pulses are accounted for. The modeled density matrix, shown in Fig. 3(b), shows very good agreement with the experimental one, and so does the purity of the modeled mixture $(0.12)$. 
(a)
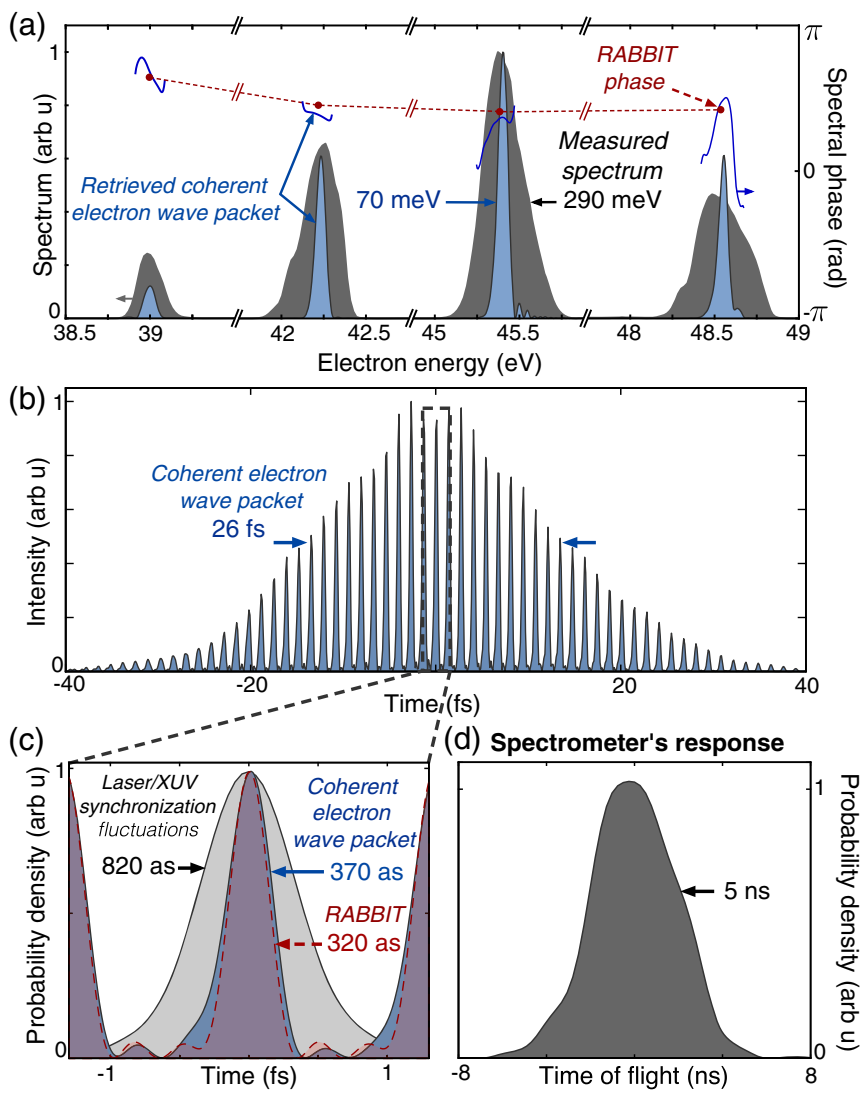

FIG. 4. Parameters of the most probable decoherence scenario. (a) Spectrum (blue shaded curve) and spectral phase (segmented blue line) of the coherent electron wave packet $\chi_{0}$ free from arrival-time variations and from the spectrometer's influence. The gray shaded curve shows the experimental spectrum also shown in Figs. 2(b) and 2(c). The red dots (connected by a dashed line) stand for the spectral phase obtained with a RABBIT measurement in the same experimental conditions except for the dressing laser intensity fixed at $4.5 \times 10^{11} \mathrm{~W} \cdot \mathrm{cm}^{-2}$. (b) Time profile $\left|\chi_{0}(t)\right|^{2}$ of the coherent electron wave packet. (c) Envelope $P$ of the fluctuations of the synchronization between the laser and XUV pulses (gray shaded curve) compared with an attosecond pulse composing the coherent electron wave packet $\chi_{0}$ (blue shaded curve) and the average pulse in the train retrieved with RABBIT (red shaded curve). (d) Response function $R$ of the time-of-flight spectrometer. All widths indicated in the figure correspond to full widths at half maximum.

Figure 4 shows the optimal parameters obtained for this model after 20 iterations of the fitting algorithm: the coherent EWP $\chi_{0}$ in Figs. 4(a) and 4(b), the arrival-time probability density $P$ in Fig. 4(c), and the spectrometer's response function $R$ in Fig. 4(d). From these results, we trace back the origin of decoherence during the experiment. A 26 fs XUV pulse train composed on average of 370 as pulses experiences arrival-time fluctuations of 820 as with respect to the laser dressing pulse. After photoionization, the EWP is detected by a spectrometer with a time-of-flight resolution of approximately $5 \mathrm{~ns}$, which is consistent with the expected resolution [50]. Importantly, it is notoriously difficult for current techniques to reconstruct a long APT [6], the limited resolution usually masking the fine details in the spectrum. By decoupling the spectrometer's response from the coherent wave packet, the present approach reveals the femtosecond structure of the train, which constitutes a dramatic improvement. For instance, the spectral phases within each harmonic [segmented blue line in Fig. 4(a)] show unexpected behaviors that deserve further investigation in the light of recent results $[19,25]$.

These results highlight the significant role of incoherent physical phenomena in attosecond experiments, even in routine conditions, and therefore raise the question of the relevance of historical techniques such as RABBIT. Compared to Mixed FROG, RABBIT evolves in a simplified picture where the EWP is considered as fully coherent and where only the averaged attosecond pulse in the train is reconstructed. For the sake of comparison, we perform a conventional RABBIT reconstruction at low dressing intensity $\left(4.5 \times 10^{11} \mathrm{~W} \cdot \mathrm{cm}^{-2}\right)$; see Figs. 4(a)-4(c). Interestingly, the attosecond pulse profile obtained with RABBIT appears to be very close to the central attosecond pulse in the coherent EWP $\chi_{0}$ retrieved earlier. Indeed, RABBIT measures only the phase difference between consecutive levels, corresponding to the phase of the terms shaded in red in Fig. 2(b), e.g., $\arg \left(\rho_{13}\right)$ or $\arg \left(\rho_{35}\right)$. As it assumes full coherence, concatenating these phase terms provides the phase difference between nonconsecutive levels, $\arg \left(\rho_{15}\right)=\arg \left(\rho_{13}\right)+\arg \left(\rho_{35}\right)$. However, it never effectively probes the higher-order coherences, which may instead be equal to zero due to decoherence, as is the case here for $\rho_{15}$. Therefore, in the present situation, we can consider that the coherent EWP obtained with RABBIT is reconstructed independently of external incoherent phenomena, explaining its close resemblance to the central peak of $\chi_{0}$.

\section{Probing complex ionization processes}

Until now, we consider photoionization mostly as an intermediate step to access the XUV pulse coherence properties. But once the beam-line decoherence has been diagnosed, minimized, and calibrated through a simple photoionization process as in the present case, the XUV pulse becomes a highly coherent well-characterized reference. By reversing the previous logic, Mixed FROG can then be exploited to probe decoherence in more complex ionization processes that are triggered by this reference. In the following, we propose to illustrate such a situation with a numerical example.

An emblematic case is that of the photoionization of the $2 s$ and $2 p$ subshells of neon. In a breakthrough experiment in 2010, Schultze et al. used an isolated XUV attosecond pulse to ionize neon atoms and measured with FROG CRAB the photoionization delay between the released $2 p$ and $2 s$ EWPs [13]. The obtained delay appeared to be much larger than expected, which marked the beginning of a long 
debate in the community. A convincing explanation was proposed in 2017 when the experiment was repeated with an APT [19]. Using narrow high harmonics instead of a broadband continuous spectrum, it was revealed that the $2 s$ contribution was quasioverlapping with a weak "shake-up" (SU) channel. As opposed to direct ionization, a SU process shares the energy of the incident photon between two electrons (from the $2 p$ subshell here), one being promoted to an upper bound state ( $3 p$ in the present case) and the second being released in the continuum with the remaining energy. It turned out that the unresolved SU channel was probably perturbing the original measurement.

This result demonstrates the crucial importance of identifying multichannel contributions in a spectrogram in order to extract meaningful information on the ionization dynamics. In the following, we show that Mixed FROG provides exactly this as well as the coherence between the different channels. We simulate a Mixed FROG measurement in the experimental conditions of Ref. [19], and we focus in particular on the interchannel coherence between the overlapping $2 s$ and SU EWPs. In this specific case, the different symmetries [53], combined with the angularly integrated measurement and the energetic distinguishability, prevent interchannel coherence. In the general case, however, coherent scenarios could emerge depending on the properties of the system, the radiation, and the detector. We thus consider two extreme scenarios: one with full coherence between the $2 s$ and SU channels and one with full decoherence. Hereafter, we refer to these two cases, respectively, as the "coherent" and "incoherent" scenarios; see Supplemental Material [42], part IV.

In this simulation, an APT ionizes neon atoms through three possible pathways: direct ionization from the $2 s$ and $2 p$ subshells and the SU process mentioned above. As the chosen APT is composed of four harmonics (H59-H65), each channel populates four energy levels of the continuum, denoted $1,3,5$, and 7 . The resulting photoelectron spectrum exhibits three replicas of the high-harmonics photon spectrum shifted in energy owing to the distinct ionization potential of each channel; see Fig. 5(a).

We now focus on the $2 s$ and SU channels. Adding a dressing laser pulse (intensity $1.5 \mathrm{TW} \cdot \mathrm{cm}^{-2}, 27$ fs FWHM, $800 \mathrm{~nm}$ ) during the ionization and varying the laser and XUV delay yields the spectrograms in Fig. 5(b) in the coherent and incoherent cases. The partial spectral overlap between the two channels induced by the IR transitions discreetly reveals the nature of the released EWP. In the incoherent case, this overlap simply reduces the contrast of the observed oscillations, whereas, in the coherent scenario, the superposition changes the structure of the beatings.

Mixed FROG is then used to process both spectrograms. Using the same inversion procedure as before, one rapidly obtains the density matrices in Fig. 5(c) in the basis of continuum energy states $\rho\left(\epsilon, \epsilon^{\prime}\right)$. Both density matrices
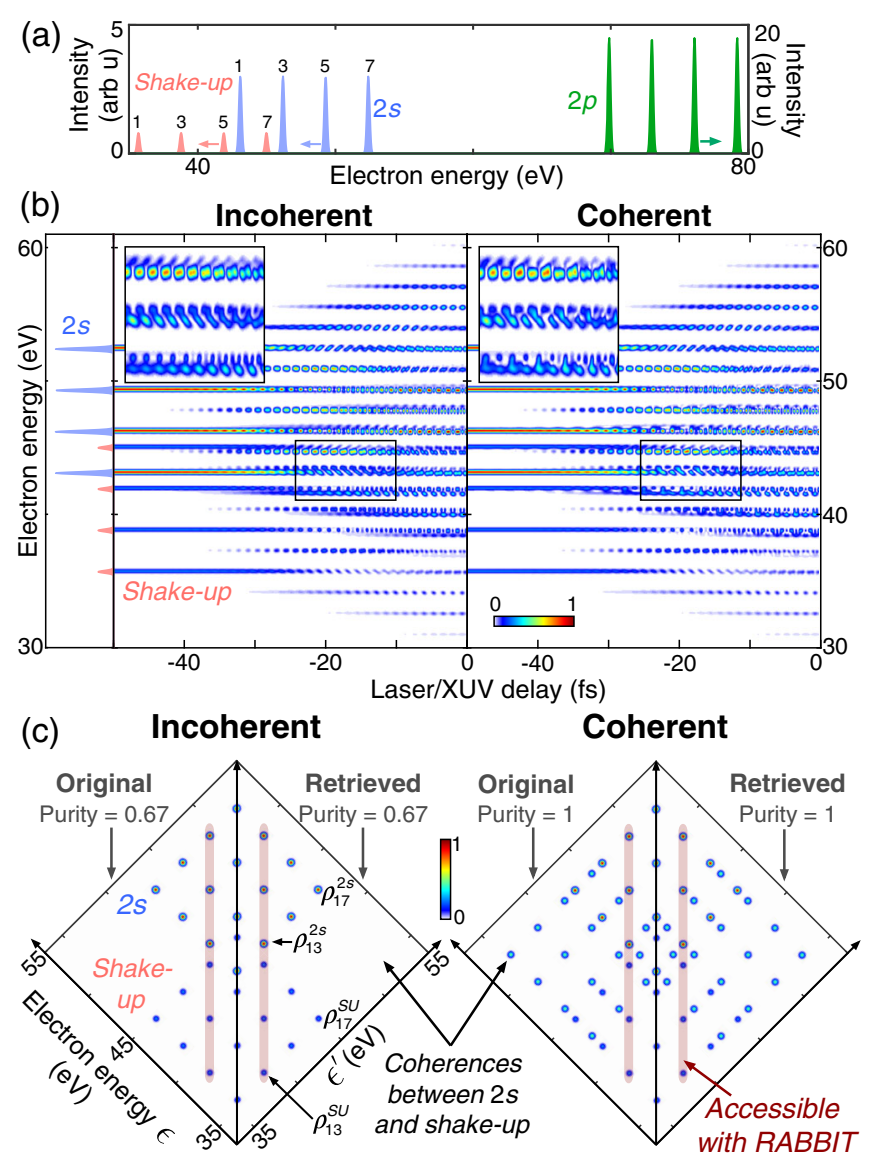

FIG. 5. Simulation of a multichannel photoelectron wave packet reconstructed with Mixed FROG. (a) Electron energy spectrum obtained after ionization of neon by high-order harmonics. The spectrum shows three ionization pathways: direct ionization from the $2 p$ and $2 s$ subshells and SU ionization; see the main text for details. (b) Simulated spectrograms in the 30-60 eV spectral region with and without interchannel coherences between the $2 s$ and SU wave packets (respectively labeled as the coherent and incoherent cases). The dressing laser pulse has an intensity of $1.5 \times 10^{12} \mathrm{~W} \cdot \mathrm{cm}^{-2}$ and a duration of $27 \mathrm{fs}$. Inset: Enlargement of the area delimited by the black square in the main panel. (c) Density matrices in the coherent and incoherent scenarios (only the modulus shown). In both cases, the left side corresponds to the original density matrix used to simulate the spectrogram in (b), and the right side shows the density matrix retrieved with Mixed FROG.

show intrachannel coherences, that is, coherences between continuum states populated through the same channel; see, for example, $\rho_{17}^{\mathrm{SU}}$ or $\rho_{17}^{2 s}$. Indeed, we consider in this simulation that the photoionization process through the $2 s$ or SU channels maintains the coherence initially present between the XUV harmonics, as observed experimentally in Fig. 2 for the $2 p$ pathway. In the coherent scenario, interchannel coherences appear. This result indicates that states populated through distinct ionization pathways, e.g., level 1 from the SU channel and level 7 from the $2 s$ channel, can interfere. Moreover, Fig. 5(c) shows that these 

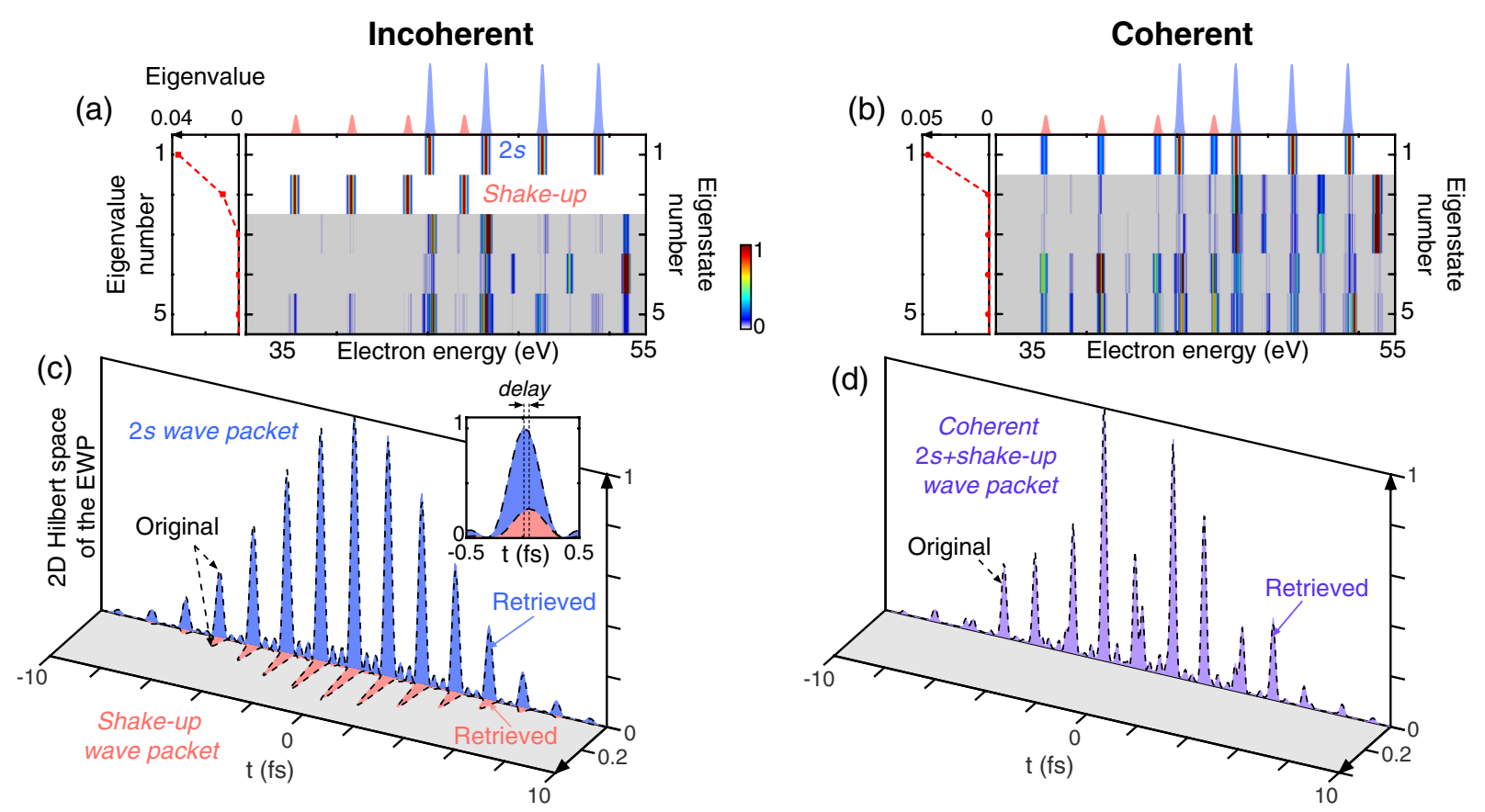

(e)

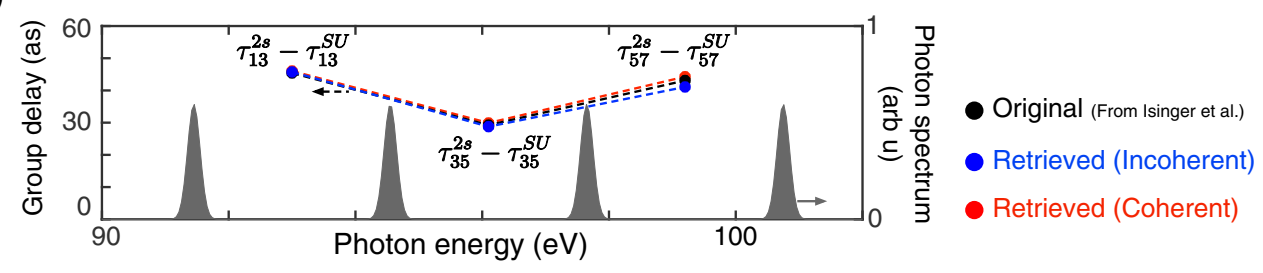

FIG. 6. Eigenstate decomposition of the multichannel density matrix in the coherent and incoherent cases. (a),(b) Eigenvalues $\lambda_{i}$ of $\rho\left(\epsilon, \epsilon^{\prime}\right)$ sorted in decreasing order (left) and modulus of the associated eigenstates $\left|\chi_{i}(\epsilon)\right|$ (right). When $\lambda_{i}$ is negligible, the eigenstate is shaded in gray. (c),(d) After Fourier transforming the dominant eigenstates, one obtains the time-domain wave packets $\left|\chi_{i}(t)\right|^{2}$ (shaded curves); the black dashed lines show the time profile of the exact wave packets used to perform the simulation. (e) The photoionization time delays can easily be recovered from the retrieved density matrices (dots connected by dashed lines); see the main text for details. The original delays are taken from Ref. [19]. The high-harmonic photon spectrum is also shown (gray shaded curve).

retrieved density matrices are in very good agreement with the original ones.

To push further the analysis, we apply the spectral theorem and decompose the retrieved density matrices into orthogonal coherent eigenstates. The results are shown in Figs. 6(a) and 6(b). In the incoherent case, two states $\chi_{i}(\epsilon)$ with non-negligible eigenvalues $\lambda_{i}$ emerge. It is straightforward to associate them to the $2 s$ and SU channels, indicating that the density matrix can be written as

$\rho\left(\epsilon, \epsilon^{\prime}\right)=\lambda_{2 s} \cdot \chi_{2 s}(\epsilon) \chi_{2 s}^{*}\left(\epsilon^{\prime}\right)+\lambda_{\mathrm{SU}} \cdot \chi_{\mathrm{SU}}(\epsilon) \chi_{\mathrm{SU}}^{*}\left(\epsilon^{\prime}\right)$.

Through this process, it becomes explicit that the wave packets from each ionization pathway belong to distinct dimensions of the multichannel EWP's Hilbert space. Conversely, in the coherent scenario, $\rho$ can be described using a single eigenstate which carries the contributions of both channels; see Fig. 6(b).

To recover the corresponding ionization dynamics, we Fourier transform the eigenstates obtained above, which provides time-domain wave packets $\chi_{i}(t)$; see Figs. 6(c) and 6(d). In the incoherent case, as each channel launches in the continuum an electronic replica of the XUV APT, the time-domain eigenstates correspond to two orthogonal trains of attosecond electron pulses. The influence of the photoionization time delays then appears as a shift of a few tens of attoseconds between the two waveforms; see the inset in Fig. 6(c). In the coherent case, the time-domain eigenstate takes the form of a complex train of attosecond pulses. The coherent superposition of the EWPs emerging from the two channels with different energies and phases creates a temporal beating in the probability density [28] that modulates the train structure. The dashed lines in Figs. 6(c) and 6(d) indicate the time profile of the original EWPs used as a starting point for this simulation. The excellent agreement with the retrieved time profiles confirms that Mixed FROG, coupled with an eigenstate decomposition of $\rho$, provides a powerful method to separate contributions from distinct channels.

More importantly, it gives access to the real ionization dynamics that may be much more intricate than the simple 
time delays $\tau_{2 s}-\tau_{\mathrm{SU}}$ may infer. Indeed, the latter just correspond to the difference in group delays between the two channels [23]; these group delays, as measured by RABBIT, are directly related to the intrachannel firstorder coherences shaded in red in Fig. 5(c) by, e.g., $\tau_{13}^{2 s}=$ $\arg \left(\rho_{13}^{2 s}\right) /\left(2 \omega_{\mathrm{IR}}\right)$ with $\omega_{\mathrm{IR}}$ the laser angular frequency. Yet these intrachannel coherences give no information on the relative coherence between the two channels. Indeed, comparing the group delays from Ref. [19] with those extracted from these coherences on the retrieved density matrices, one obtains identical photoionization delays in the coherent and incoherent cases. This result highlights that the quantum behavior of a multichannel EWP cannot, in general, be summarized to a group delay and should instead be considered through its density matrix.

\section{CONCLUSION}

Mixed FROG constitutes a powerful diagnostic for attosecond metrology. The technique that we demonstrate experimentally in the case of an attosecond pulse train can, in principle, reconstruct any waveform structure. When combined with decoherence modeling, it enables the reconstructed EWP to become, up to a certain extent, independent of the detector resolution and of inaccuracies in the synchronization between the laser and XUV pulses. Therefore, this technique could open the way to superresolution photoelectron spectroscopy and jitter-free experiments with ultrashort XUV sources. Moreover, by enabling the analysis of complete attosecond pulse trains, it could contribute to refine our understanding of the highharmonic generation process. Mixed FROG could also benefit the rapidly growing field of attosecond electron microscopy [54] by enabling the complete phase-space characterization of ultrashort relativistic electron bunches. This characterization would allow one to probe samples with attosecond and sub-Angström resolutions.

This approach is also full of promise for the study of decoherence originating from photoionization itself. Reconstructing the full quantum state of photoelectron wave packets would give access to a physics much more subtle than photoionization time delay measurements, as illustrated here in the case of multichannel ionization. Moreover, with the current trend to decrease the duration of attosecond sources and, thus, to broaden the XUV spectrum, attosecond photoionization experiments will naturally involve more and more electronic shells, making routine the creation of multichannel EWPs. In this context, an important improvement will be to adapt Mixed FROG to account for the exact influence of the laser field on broadband EWPs as has been done in the fully coherent case $[8,9]$.

For the past 60 years, photoemission spectroscopy has been one of the flagship techniques for studying the electronic structure of matter. Recent measurements of photoionization coherent dynamics have lifted the veil on the chronology of the photoelectric effect at the femtosecond and attosecond timescales [25,55]. By quantifying decoherence in photoemission, Mixed FROG will extend the scope of attosecond spectroscopy to a broad range of partially coherent quantum processes $[24,26]$.

\section{ACKNOWLEDGMENTS}

We gratefully thank our colleague and friend Bertrand Carré for his continuous support and visionary insight into attosecond science. This research received the financial support of the French National Research Agency through Grants No. ANR-14-CE32-0010-XSTASE, No. ANR-15CE30-0001-CIMBAAD, No. ANR-11-EQPX0005ATTOLAB, and No. ANR-10-LABX-0039-PALM, as well as the support of the European Union Grants No. EU-H2020MSCA-ITN-641789-MEDEA and No. EU-H2020-654148Laserlab-Europe.

\section{APPENDIX A: REPRESENTATION OF THE ELECTRON WAVE PACKET'S QUANTUM STATE}

\section{Single-electron wave packet}

To describe photoionization at the scale of a singleelectron wave packet, we build upon the theoretical framework derived in Ref. [11]. The single-photon ionization of an atom by the XUV field frees a single-electron wave packet (SEWP) in a state $|\psi\rangle$ in the continuum. Within the first-order perturbation theory and with the dipole and rotating-wave approximations, the energydomain wave packet at a distance $x$ from the atom is defined as

$$
\tilde{\chi}_{x}\left(\epsilon=\frac{p^{2}}{2}\right)=\langle\epsilon \mid \psi\rangle=-i D(p) E\left(\omega-I_{p}\right) e^{i x p},
$$

where $p$ denotes the asymptotic momentum of the SEWP in the observation direction, $\epsilon$ the corresponding kinetic energy, and $E(\omega)$ the complex XUV electric field in the angular frequency domain. $\omega$ and $\epsilon$ are related through the equation $\omega=\epsilon+I_{p}$ in atomic units, with $I_{p}$ the ionization potential of the atom. $D(p)$ represents the dipole transition matrix element of the ionized atomic species. $x$ is assumed to be sufficiently large so that the SEWP no longer feels the ionic potential and can be considered as propagating in free space. We also refer to its Fourier counterpart $\chi_{x}(t)$ as the time-domain wave packet at position $x$ :

$$
\chi_{x}(t)=\frac{1}{2 \pi} \int_{-\infty}^{+\infty} \tilde{\chi}_{x}(\epsilon) e^{-i \epsilon t} d \epsilon .
$$

To describe the SEWP in phase space, the most natural set of quadratures is the famous $[x, p]$ inherited from the harmonic oscillator. However, as free-space propagation 
couples longitudinal position $x$ and time $t$, these two quantities can be equivalently chosen to form a set of quadratures in combination with $p$ or $\epsilon$. With the $[\epsilon, t]$ set of quadratures, the Wigner distribution reads

$\mathcal{W}_{x}(\epsilon, t)=\frac{1}{2 \pi} \int_{-\infty}^{+\infty} \tilde{\chi}_{x}\left(\epsilon-\frac{\epsilon^{\prime}}{2}\right) \tilde{\chi}_{x}^{*}\left(\epsilon+\frac{\epsilon^{\prime}}{2}\right) e^{-i \epsilon^{\prime} t} d \epsilon^{\prime}$.

According to the well-known properties of the Wigner distribution, the marginal distribution $\int \mathcal{W}_{x}(\epsilon, t) d t$ gives the energy spectrum $\left|\tilde{\chi}_{x}(\epsilon)\right|^{2}$, i.e., the density of the energy states occupied by the SEWP in the continuum. Similarly, $\left|\chi_{x}(t)\right|^{2}=\int \mathcal{W}_{x}(\epsilon, t) d \epsilon$ corresponds to the time profile of the SEWP, that is, the probability of observing the electron at position $x$ and time $t$.

As it propagates in free space, the SEWP stretches in time. Experimentally, the detector can observe the evolution of the wave packet only at macroscopic times and distances. We denote $S(t)$ the time profile of the wave packet at the distance $x=L$ from the interaction region:

$$
\begin{aligned}
S(t) & =\left|\chi_{x=L}(t)\right|^{2} \\
& =\left|\frac{1}{2 \pi} \int_{-\infty}^{+\infty} \tilde{\chi}_{x=0}(\epsilon) e^{i L \sqrt{2 \epsilon}-i \epsilon t} d \epsilon\right|^{2} .
\end{aligned}
$$

When this distance is large enough, the stretching of the SEWP becomes so important that the time profile $S$ maps the energy profile. Indeed, applying the stationary phase approximation in Eq. (A4), it comes that for large phase terms, that is, for $|L \sqrt{2 \epsilon}-\epsilon t| \gg 2 \pi$, the integral is zero except for the times of flight $t_{f}$ such that $d\left(L \sqrt{2 \epsilon}-\epsilon t_{f}\right) / d \epsilon=0:$

$$
S\left(t_{f}\right) \propto \epsilon\left(t_{f}\right)^{3 / 2} \cdot\left|\tilde{\chi}_{x=0}\left[\epsilon\left(t_{f}\right)\right]\right|^{2},
$$

where $t_{f}$ and $\epsilon$ are related through the formula $\epsilon\left(t_{f}\right)=$ $1 / 2 \cdot\left(L / t_{f}\right)^{2}$. This regime is used in TOF spectrometry. The term $\tilde{\chi}_{x=0}(\epsilon)$ corresponds to the energy-domain wave packet defined in Eq. (A1) backpropagated in free space to its point of origin in $x=0$. However, it does not physically represent the wave packet that effectively exists near the atom at the ionization time and which instead propagates in the ionic potential. $\chi_{x=0}$ thus describes a hybrid SEWP which possesses its asymptotic momentum distribution but does not exhibit temporal stretching due to free-space propagation. It is this wave packet that is actually characterized in the following; therefore, we now discard the subscript $x=0$ in the notation for clarity.

\section{Macroscopic electron wave packet and mixed-state formalism}

The quantity measured by the electron spectrometer is the averaged TOF distribution $\left\langle S\left(t_{f}\right)\right\rangle$. The averaging accounts for all the degrees of freedom that remain unresolved in the experiment. Some of these phenomena are exemplified in Appendix B. The macroscopic electron wave packet (MEWP) that is built from all the detected electrons needs to be described as a statistical mixture of SEWPs through the density matrix formalism. In this representation, the averaging \langle\rangle of the macroscopic quantities is rewritten as a weighted sum over a discrete number of states which are mutually incoherent. Consider the density matrix $\rho=\sum_{k} p_{k}\left|\psi_{k}\right\rangle\left\langle\psi_{k}\right|$, where $\left\{\left|\psi_{k}\right\rangle\right\}_{k}$ forms an orthonormal basis of states composing the mixture and $p_{k}$ is the probability of observing each state with $\sum_{k} p_{k}=1$. In the energy domain, the density matrix elements read

$$
\begin{aligned}
\rho\left(\epsilon, \epsilon^{\prime}\right) & =\left\langle\epsilon|\rho| \epsilon^{\prime}\right\rangle \\
& =\sum_{k} p_{k} \cdot\left\langle\epsilon \mid \psi_{k}\right\rangle\left\langle\psi_{k} \mid \epsilon^{\prime}\right\rangle=\sum_{k} p_{k} \cdot \tilde{\chi}_{k}(\epsilon) \tilde{\chi}_{k}^{*}\left(\epsilon^{\prime}\right),
\end{aligned}
$$

where $\tilde{\chi}_{k}(\epsilon)$ denotes the energy-domain wave packets composing the mixture. Rewriting Eq. (A5) in the mixed state formalism gives

$$
\left\langle S\left(t_{f}\right)\right\rangle \propto \epsilon^{3 / 2} \sum_{k} p_{k}\left|\tilde{\chi}_{k}(\epsilon)\right|^{2}=\epsilon^{3 / 2} \cdot \rho(\epsilon, \epsilon) .
$$

Measuring the TOF distribution of the MEWP provides the diagonal elements of the density matrix in energy representation, that is, the energy state populations. The off-diagonal elements $\rho\left(\epsilon, \epsilon^{\prime}\right)$ with $\epsilon \neq \epsilon^{\prime}$, the coherences, could be deduced from the hypothetical interference between two energy states at energy $\epsilon$ and $\epsilon^{\prime}$ : $\sum_{k} p_{k} \cdot\left|\left\langle\epsilon \mid \psi_{k}\right\rangle+\left\langle\epsilon^{\prime} \mid \psi_{k}\right\rangle\right|^{2}=\rho(\epsilon, \epsilon)+\rho\left(\epsilon^{\prime}, \epsilon^{\prime}\right)+2\left|\rho\left(\epsilon, \epsilon^{\prime}\right)\right| \times$ $\cos \left[\arg \left\{\rho\left(\epsilon, \epsilon^{\prime}\right)\right\}\right]$, where $\arg (z)$ denotes the argument of the complex number $z$. Equivalently, we define the timedomain density matrix elements as

$$
\begin{aligned}
\rho\left(t, t^{\prime}\right) & =\frac{1}{(2 \pi)^{2}} \iint \rho\left(\epsilon, \epsilon^{\prime}\right) e^{-i\left(\epsilon t-\epsilon^{\prime} t^{\prime}\right)} d \epsilon d \epsilon^{\prime} \\
& =\sum_{k} p_{k} \cdot \chi_{k}(t) \chi_{k}^{*}\left(t^{\prime}\right) .
\end{aligned}
$$

The diagonal elements now correspond to the average time profile of the SEWPs in the mixture $\rho(t, t)=$ $\sum_{k} p_{k}\left|\chi_{k}(t)\right|^{2}$, whereas the off-diagonal elements give the interference term between the MEWP at time $t$ and itself at time $t^{\prime}$. The overall coherence of the statistical ensemble of SEWPs can be quantified by estimating the purity $\nu$ defined as

$$
\begin{aligned}
\nu & =\operatorname{Tr}\left(\rho^{2}\right)=\iint\left|\rho\left(\epsilon, \epsilon^{\prime}\right)\right|^{2} d \epsilon d \epsilon^{\prime} \\
& =(2 \pi)^{2} \iint\left|\rho\left(t, t^{\prime}\right)\right|^{2} d t d t^{\prime},
\end{aligned}
$$

with $\operatorname{Tr}$ the trace operator. The purity equals one if all the SEWPs are in the same state; otherwise, the MEWP is in a mixed state and $\nu<1$. 
To complete this new formalism, the definition of the Wigner distribution can be updated in the case of mixed states and related to the density matrix by the formula

$$
\begin{aligned}
W(\epsilon, t) & =\sum_{k} p_{k} \mathcal{W}_{k}(\epsilon, t) \\
& =\frac{1}{2 \pi} \int \rho\left(\epsilon-\frac{\epsilon^{\prime}}{2}, \epsilon+\frac{\epsilon^{\prime}}{2}\right) e^{-i \epsilon^{\prime} t} d \epsilon^{\prime} \\
& =\int \rho\left(t-\frac{t^{\prime}}{2}, t+\frac{t^{\prime}}{2}\right) e^{i \epsilon t^{\prime}} d t^{\prime} .
\end{aligned}
$$

The density matrix, in any basis, and the Wigner distribution are related by bijective relationships and, therefore, equivalently describe the quantum state of the MEWP. It is this quantity that one wants to reconstruct.

\section{Influence of the laser field}

When no external coherent laser field is present, the photoelectron kinetic energy spectrum can be expressed in various forms:

$$
\begin{aligned}
\langle S(\epsilon)\rangle & =\sum_{k} p_{k}\left|\int \chi_{k}(t) e^{i \epsilon t} d t\right|^{2} \\
& =\sum_{k} p_{k}\left|\left\langle\epsilon \mid \psi_{k}\right\rangle\right|^{2} \\
& =\langle\epsilon|\rho| \epsilon\rangle .
\end{aligned}
$$

Consider that a laser pulse with a vector potential $A(t)$ is synchronized at a delay $\tau$ with the XUV pulse that triggers photoionization. The XUV and laser pulses are linearly polarized with collinear polarization axes, and the MEWP is observed along this axis. In the frame of the first-order perturbation theory with the single active electron approximation, one can extend to the case of state mixtures the well-established formula giving the photoelectron spectrum in the presence of the laser pulse:

$$
\begin{aligned}
\langle S(\epsilon, \tau)\rangle & =\sum_{k} p_{k}\left|\int \chi_{k}(t) G(t-\tau) e^{i \epsilon t} d t\right|^{2} \\
& =\sum_{k} p_{k}\left|\left\langle\epsilon\left|\hat{G}_{\tau}\right| \psi_{k}\right\rangle\right|^{2},
\end{aligned}
$$

where $G(t)=e^{i \phi(t)}$ and where $\phi(t)=-\int_{t}^{+\infty}\left[p A\left(t^{\prime \prime}\right)+\right.$ $\left.A^{2}\left(t^{\prime \prime}\right) / 2\right] d t^{\prime \prime}$ is the phase modulator [11]. In the FROG framework, the momentum $p$ is often approximated by $p_{0}$ the central momentum of the MEWP, $\chi_{k}(t)$ is referred to as the pulse and $G$ as the gate, and Eq. (A12) represents an incoherent sum of spectrograms. The influence of the gate can also be described as an operator $\hat{G}_{\tau}$ acting on the electron wave packet.

\section{Influence on the density matrix}

In order to introduce the density operator in the above equation, we now assume that $G$ is a fully coherent phase modulator, that is, that each SEWP experiences the same laser vector potential. This assumption enables the following factorization:

$$
\begin{aligned}
\langle S(\epsilon, \tau)\rangle & =\sum_{k} p_{k}\left\langle\epsilon\left|\hat{G}_{\tau}\right| \psi_{k}\right\rangle\left\langle\psi_{k}\left|\hat{G}_{\tau}^{\dagger}\right| \epsilon\right\rangle \\
& =\left\langle\epsilon\left|\hat{G}_{\tau} \rho \hat{G}_{\tau}^{\dagger}\right| \epsilon\right\rangle,
\end{aligned}
$$

where $\hat{G}_{\tau}^{\dagger}$ denotes the Hermitian conjugate of $\hat{G}_{\tau}$. In the presence of a dressing laser field, the kinetic energy spectrum corresponds to the diagonal elements of a modified density matrix $\rho^{\prime}=\hat{G}_{\tau} \rho \hat{G}_{\tau}^{\dagger}$ [37]. By this process, the off-diagonal elements of $\rho$ become encoded into the populations of $\rho^{\prime}$, which thus gives access to the full laserfree density matrix $\rho$.

\section{Influence on the Wigner distribution}

In the absence of an external laser field, the measured spectrum corresponds to the projection of the Wigner distribution of the MEWP along the kinetic energy axis:

$$
\langle S(\epsilon)\rangle=\sum_{k} p_{k} \int \mathcal{W}_{k}(\epsilon, t) d t=\int W(\epsilon, t) d t
$$

The Wigner distribution cannot be deduced from this sole projection. The role of the laser field is therefore to operate a transformation on $W(\epsilon, t)$ before projecting it onto the energy axis, thus giving access to extra information on $W$. If the laser field is present, $\langle S(\epsilon, \tau)\rangle$ reads as an incoherent sum of pure spectrograms [see Eq. (A12)], and each of them can be expressed as the convolution between the Wigner distribution of the pure wave packet $\mathcal{W}_{k}(\epsilon, t)$ and that of the gate $W_{G}(\epsilon, t)[56]$ :

$$
\begin{aligned}
\langle S(\epsilon, \tau)\rangle & =\sum_{k} p_{k}\left|\int \chi_{k}(t) G(t-\tau) e^{i \epsilon t} d t\right|^{2} \\
& =\sum_{k} p_{k} \iint \mathcal{W}_{k}(\delta \epsilon, t) W_{G}(\epsilon-\delta \epsilon, t-\tau) d t d \delta \epsilon,
\end{aligned}
$$

with $W_{G}(\epsilon, t)=\int G\left(t-t^{\prime} / 2\right) G^{*}\left(t+t^{\prime} / 2\right) e^{i \epsilon t^{\prime}} d t^{\prime}$. As in the case of Eq. (A13), if the gate is a coherent phase modulator, $W_{G}$ factorizes out, which enables the emergence of the Wigner distribution of the MEWP:

$$
\begin{aligned}
\langle S(\epsilon, \tau)\rangle & =\iint W(\delta \epsilon, t) W_{G}(\epsilon-\delta \epsilon, t-\tau) d t d \delta \epsilon \\
& =\int W^{\prime}(\epsilon, t) d t .
\end{aligned}
$$

$W^{\prime}(\epsilon, t)$ corresponds to the new Wigner distribution of the MEWP after it has been modified by the gate. In the presence of the laser field, the kinetic energy spectrum thus 
represents the projection of $W^{\prime}(\epsilon, t)$ onto the energy axis. As the influence of the gate changes with $\tau$, the final spectrogram can be thought of as a series of projections of $W^{\prime}(\epsilon, t)$. The process of quantum state tomography consists in reconstructing $W(\epsilon, t)$ from these projections.

\section{APPENDIX B: IDENTIFYING THE EXPERIMENTAL SOURCES OF DECOHERENCE}

To understand the origin of the observed decoherence, we carry out the following study. First, we identify the potential sources of decoherence in the conditions of the experiment and model their impact mathematically. Then, we fit these modeled density matrices in the least-squares sense onto the experimental one to find the model that best describes the data.

By combining Eqs. (A1) and (A6), the role of the XUV electric field $E$ in the density matrix in the energy domain appears explicitly:

$$
\begin{aligned}
\rho\left(\epsilon, \epsilon^{\prime}\right) & =\sum_{k} p_{k} \cdot \tilde{\chi}_{k}(\epsilon) \tilde{\chi}_{k}^{*}\left(\epsilon^{\prime}\right) \\
& =\sum_{k} p_{k} \cdot D(\sqrt{2 \epsilon}) E_{k}\left(\omega-I_{p}\right) \cdot D^{*}\left(\sqrt{2 \epsilon^{\prime}}\right) E_{k}^{*}\left(\omega^{\prime}-I_{p}\right) \\
& =\gamma_{\mathrm{at}}\left(\epsilon, \epsilon^{\prime}\right) c\left(\epsilon, \epsilon^{\prime}\right),
\end{aligned}
$$

where $c\left(\omega, \omega^{\prime}\right)=\sum_{k} p_{k} \cdot E_{k}(\omega) E_{k}^{*}\left(\omega^{\prime}\right)$ is the twofrequency correlation function of the XUV pulse ensemble. We here consider that the transition dipole matrix element $D$ is identical for all the wave packets of the mixture and define the atomic function $\gamma_{\mathrm{at}}\left(\epsilon, \epsilon^{\prime}\right)=D(\sqrt{2 \epsilon}) D^{*}\left(\sqrt{2 \epsilon^{\prime}}\right)$. In that case, Eq. (B1) states that the coherence state of the XUV pulse ensemble described by $c\left(\omega, \omega^{\prime}\right)$ directly influences the EWP quantum state. Moreover, in the present experimental study, the released wave packet lies in the structureless area of neon continuum, so that $\gamma_{\text {at }}$ can be considered as independent of $\epsilon$ and $\epsilon^{\prime}$ in practice.

\section{XUV partial coherence}

Partial coherence of the XUV pulse ensemble can arise from any variation of the XUV electric field in unresolved degrees of freedom, such as the transverse spatial structure and the shot-to-shot structure of the pulses. We estimate that one of the most relevant sources of decoherence for the XUV pulse ensemble corresponds to arrival-time variations. If the XUV beam is slightly out of focus in the gas jet, or, more generally, if it suffers from optical aberrations, the pulse ensemble takes the form of a group of a priori identical XUV pulses with different arrival times. Importantly, this arrival time is meaningful only when compared to that of the IR-dressing pulse. Therefore, mechanical instabilities in the laser dressing beam path could lead to a similar XUV pulse ensemble.

Its impact on the density matrix can be modeled in the following way. The ensemble of XUV pulses is composed of attosecond pulse trains $E_{0}(\omega) e^{i\left(\omega-\omega_{0}\right) T}$ of central frequency $\omega_{0}$ arriving at different times $T$, the range of arrival times being determined by the probability density $P(T)$. Rewriting Eq. (B1) in this scenario gives

$$
\begin{aligned}
& \rho_{\text {model }}\left(\epsilon, \epsilon^{\prime}\right) \\
& =\gamma_{\text {at }} \int E_{0}(\epsilon) e^{i\left(\epsilon-\omega_{0}\right) T} E_{0}^{*}\left(\epsilon^{\prime}\right) e^{-i\left(\epsilon^{\prime}-\omega_{0}\right) T} P(T) d T \\
& =\tilde{\chi}_{0}(\epsilon) \tilde{\chi}_{0}^{*}\left(\epsilon^{\prime}\right) \int P(T) e^{i\left(\epsilon-\epsilon^{\prime}\right) T} d T,
\end{aligned}
$$

with $\tilde{\chi}_{0}(\epsilon)=-i D(\sqrt{2 \epsilon}) E_{0}(\epsilon)$. Introducing $\mathcal{P}(\omega)$, the Fourier transform of $P(t)$, Eq. (B2) becomes

$$
\rho_{\text {model }}\left(\epsilon, \epsilon^{\prime}\right)=\tilde{\chi}_{0}(\epsilon) \cdot \tilde{\chi}_{0}^{*}\left(\epsilon^{\prime}\right) \cdot \mathcal{P}\left(\epsilon-\epsilon^{\prime}\right) .
$$

The high-harmonic generation mechanism can lead to a transverse variation of the attochirp across the XUV beam [47]. In this case, the XUV pulse ensemble is composed of waveforms $E_{0}(\omega) e^{i\left(\omega-\omega_{0}\right)^{2} \alpha}$ experiencing variable group delay dispersion $2 \alpha$. If denoting $C(\alpha)$ the associated probability density and $\mathcal{C}$ its Fourier transform, a calculation similar to Eqs. (B2) and (B3) gives

$\rho_{\text {model }}\left(\epsilon, \epsilon^{\prime}\right)=\tilde{\chi}_{0}(\epsilon) \cdot \tilde{\chi}_{0}^{*}\left(\epsilon^{\prime}\right) \cdot \mathcal{C}\left[\left(\epsilon-\epsilon_{0}\right)^{2}-\left(\epsilon^{\prime}-\epsilon_{0}\right)^{2}\right]$

with $\epsilon_{0}=\omega_{0}-I_{p}$. In both cases, the mixed state can thus be modeled as a pure density matrix $\tilde{\chi}_{0}(\epsilon) \cdot \tilde{\chi}_{0}^{*}\left(\epsilon^{\prime}\right)$ multiplied with an extra decoherence term depending on $\mathcal{P}$ or $\mathcal{C}$.

We illustrate the impact of these sources of decoherence in Fig. 7. A statistical ensemble of attosecond pulse trains composed of five odd harmonics of a laser at $800 \mathrm{~nm}$ ionizes $\mathrm{Ne}$ atoms, which populates five states of the continuum. In Fig. 7(a), the XUV pulses composing the ensemble are identical, the resulting density matrix is pure, and no alteration of its off-diagonal elements is observed. In Fig. 7(b), we account for arrival-time variations. The XUV pulses can experience random delays $T$ within an envelope $P(T)$ that is Gaussian with a full width at half maximum (FWHM) of 450 as. One can notice the disappearance of some off-diagonal elements, the highest-order coherences being the most affected. Finally, in Fig. 7(c), the attosecond pulses are perfectly synchronized, but their group delay dispersion can vary within a Gaussian envelope centered in $\alpha=0 \mathrm{as}^{2} / \mathrm{rad}$ with a FWHM of $8 \times 10^{5} \mathrm{as}^{2} / \mathrm{rad}$. The coherences are this time attenuated in a different way.

\section{Collisional decoherence}

As the SEWPs propagate toward the detector $(0<x<L)$, they can collide with atoms of neon from the gas jet. The gas density in the jet is estimated to be $10^{12} \mathrm{~cm}^{-3} \leq n_{\mathrm{at}} \leq 10^{14} \mathrm{~cm}^{-3}$, and the electron-neon 


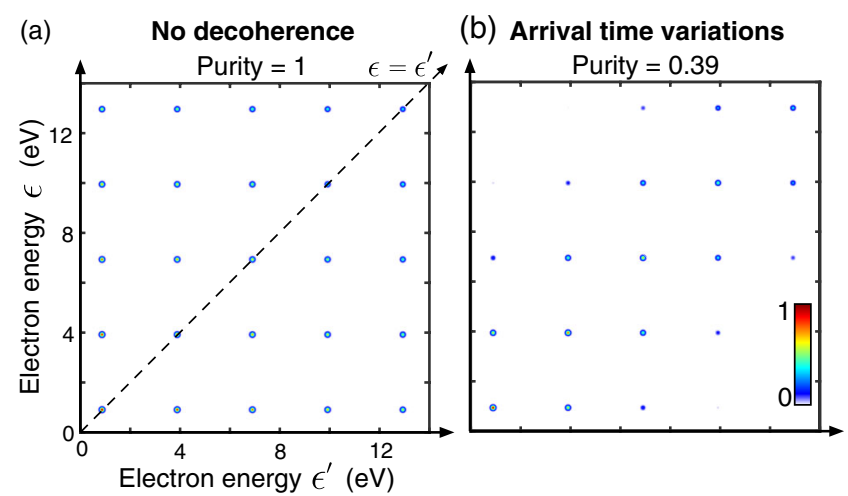

(c)

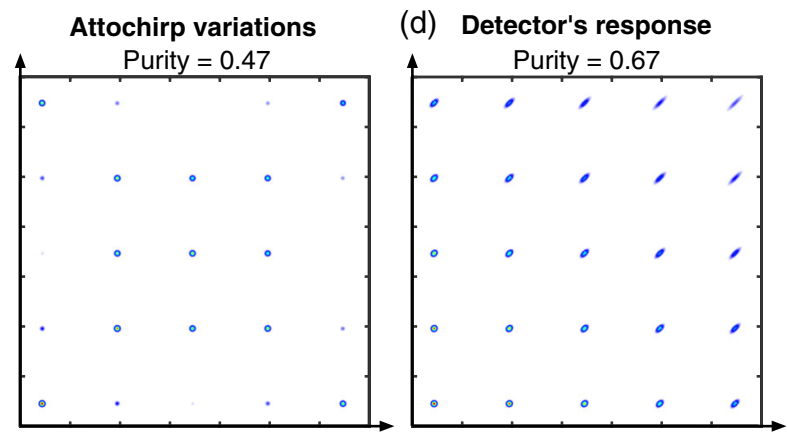

FIG. 7. Simulated density matrices in various cases. (a) The electron wave packet is pure, (b) the XUV pulses suffer from arrival-time variations, or (c) attochirp variations. (d) The XUV pulse ensemble is fully coherent, but the limited resolution of the spectrometer is accounted for.

elastic scattering total cross section for electrons at $50 \mathrm{eV}$ is $\sigma=3.1 \times 10^{-16} \mathrm{~cm}^{2}$ [57]. In these conditions, the mean free path $1 /\left(\sigma \cdot n_{\text {at }}\right)$ of the electrons is comprised between $22 \mathrm{~cm}$ and $22 \mathrm{~m}$. This result is obviously orders of magnitude larger than the size of the gas jet. Therefore, we estimate that collisional decoherence should be negligible.

\section{Detection device}

The last potential source of decoherence in our experimental conditions is the limited temporal resolution of the TOF spectrometer. This apparatus measures the arrival time $t_{f}$ of the electrons (in the nanosecond or microsecond range) after traveling the distance $L$ that separates the interaction region from the detector. Without external laser modulation, the TOF distribution can be related to the Wigner distribution of the EWP by $\left\langle S\left(t_{f}\right)\right\rangle=t_{f}^{-3} \int W\left[t, \epsilon\left(t_{f}\right)\right] d t$, with $\epsilon\left(t_{f}\right)=1 / 2 \cdot\left(L / t_{f}\right)^{2}$. Using Eq. (A16), an equivalent relation can be obtained when a laser pulse with a delay $\tau$ is present:

$$
\left\langle S\left(t_{f}, \tau\right)\right\rangle=t_{f}^{-3} \iint W\left[t, \epsilon\left(t_{f}\right)-\delta \epsilon\right] \cdot W_{G}(t-\tau, \delta \epsilon) d t d \delta \epsilon .
$$

With an ideal detector, this equation provides the signal that is effectively measured. And the algorithm described above separates the contribution from the gate and from the pulse mixture to retrieve $W$. However, in practice, the TOF resolution of the spectrometer may be limited by a variety of phenomena (the size of the interaction region, the various trajectories of the collected electrons, the response time of the microchannel plates, the bandwidth of the data acquisition system, etc.). We model this influence through the convolution of the detector-free TOF signal $\left\langle S_{0}\right\rangle$ with $R$, a probability density representing the TOF response of the detector, such that $\left\langle S\left(t_{f}\right)\right\rangle=\int R\left(\delta t_{f}\right)\left\langle S_{0}\left(t_{f}-\delta t_{f}\right)\right\rangle d \delta t_{f}$. Inserting this equation into Eq. (B5), one gets

$$
\begin{aligned}
\left\langle S\left(t_{f}, \tau\right)\right\rangle & =\int d \delta t_{f} \cdot R\left(\delta t_{f}\right) \cdot\left(t_{f}-\delta t_{f}\right)^{-3} \iint W\left[t, \epsilon\left(t_{f}-\delta t_{f}\right)-\delta \epsilon\right] \cdot W_{G}(t-\tau, \delta \epsilon) d t d \delta \epsilon \\
& =t_{f}^{-3} \iint W_{\text {detected }}\left[t, \epsilon\left(t_{f}\right)-\delta \epsilon\right] \cdot W_{G}(t-\tau, \delta \epsilon) d t d \delta \epsilon,
\end{aligned}
$$

with $W_{\text {detected }}(t, \epsilon)=\int\left[R\left(\delta t_{f}\right) /\left(1-\delta t_{f} / t_{f}\right)^{3}\right] \cdot W\left[t, \epsilon\left(t_{f}-\right.\right.$ $\left.\left.\delta t_{f}\right)\right] d \delta t_{f}$. This time, the algorithm reconstructs a new state $W_{\text {detected }}$ affected by the detector's response function. As $W_{\text {detected }}$ reads as a weighted sum of Wigner distributions, the detector's influence appears as a loss of coherence of the reconstructed state. Interestingly, this decoherence process is contained in the reconstructed state even though the detection step technically occurs well after the laser pulse probes the EWP. Finally, the density matrix model associated to $W_{\text {detected }}$ is obtained; for convenience, we use center- and difference-energy variables $\epsilon_{c}=\left(\epsilon+\epsilon^{\prime}\right) / 2$ and $\Delta \epsilon=\epsilon^{\prime}-\epsilon$ :

$$
\begin{aligned}
\rho_{\text {model }}\left(\epsilon_{c}, \Delta \epsilon\right) & =2 \pi \int W_{\text {detected }}\left(t, \epsilon_{c}\right) e^{i \Delta \epsilon t} d \Delta \epsilon \\
& =\int \frac{R\left(\delta t_{f}\right)}{\left(1-\delta t_{f} / t_{f}\right)^{3}} \cdot \rho_{\mathrm{df}}\left[\epsilon_{c}\left(t_{f}-\delta t_{f}\right)-\frac{\Delta \epsilon}{2}, \epsilon_{c}\left(t_{f}-\delta t_{f}\right)+\frac{\Delta \epsilon}{2}\right] d \delta t_{f},
\end{aligned}
$$


where $\rho_{\mathrm{df}}$ is the detector-free density matrix. Using Eq. (B7), we simulate the effect of the detector's response on the density matrix; see Fig. 7(d). $\rho_{\mathrm{df}}$ is chosen to be pure, i.e., $\rho_{\mathrm{df}}\left(\epsilon, \epsilon^{\prime}\right) \propto \tilde{\chi}_{0}(\epsilon) \cdot \tilde{\chi}_{0}^{*}\left(\epsilon^{\prime}\right)$, and the parameters for the attosecond pulse train are the same as in Fig. 7(a). In this simulation, the detector's response $R\left(\delta t_{f}\right)$ is a Gaussian probability density with a FWHM of 14 ns. In these conditions, we notice that the coherences and populations appear to be stretched along the $\epsilon=\epsilon^{\prime}$ axis, and this elongation effect increases with the central energy $\left(\epsilon+\epsilon^{\prime}\right) / 2$. This result makes sense, since the energy resolution of TOF spectrometers worsens as the energy increases. Therefore, instead of reproducing the group of equally

(a)

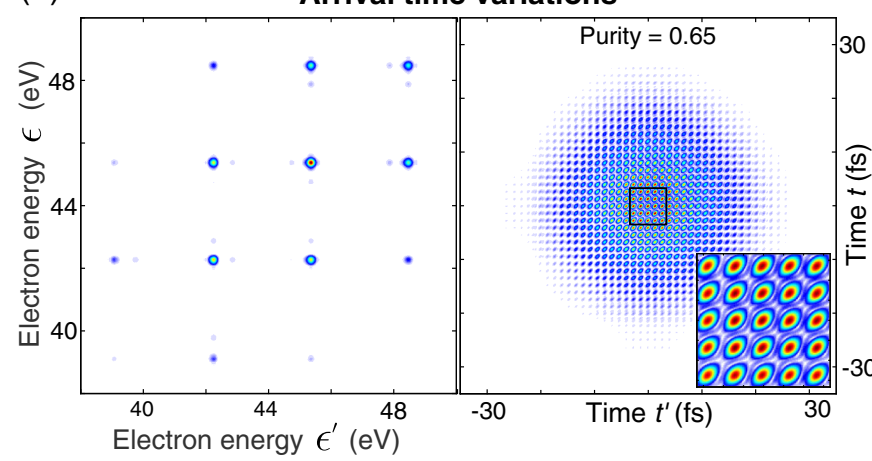

(c)

Spectrometer's response
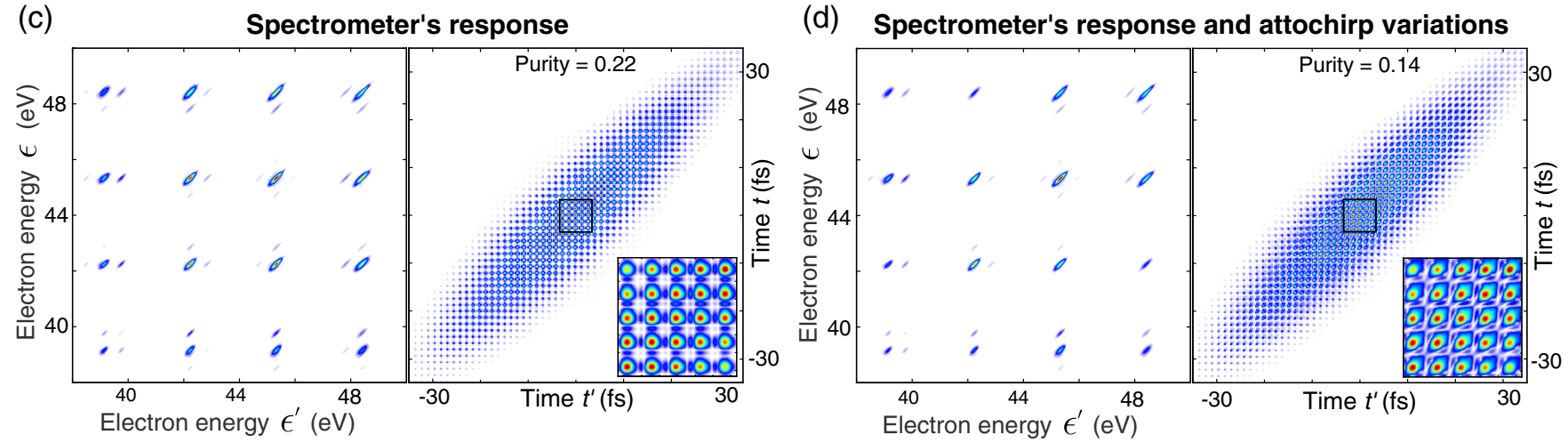

(e) Spectrometer's response and arrival time variations

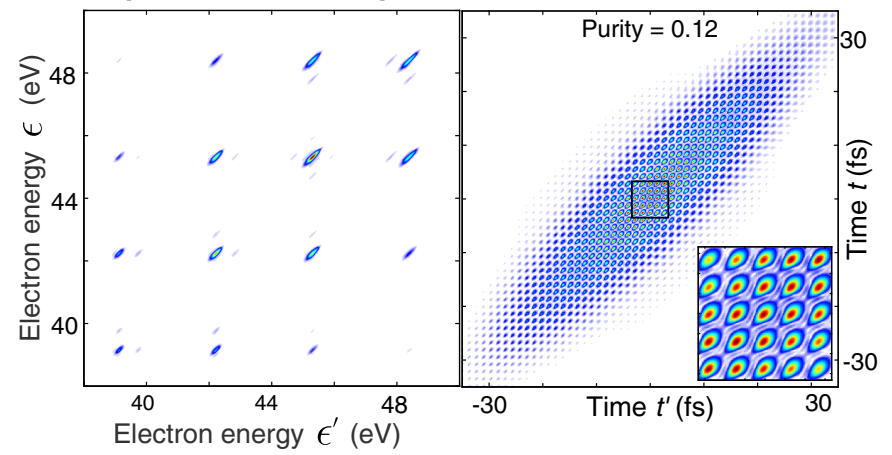

(f) Experimental density matrix

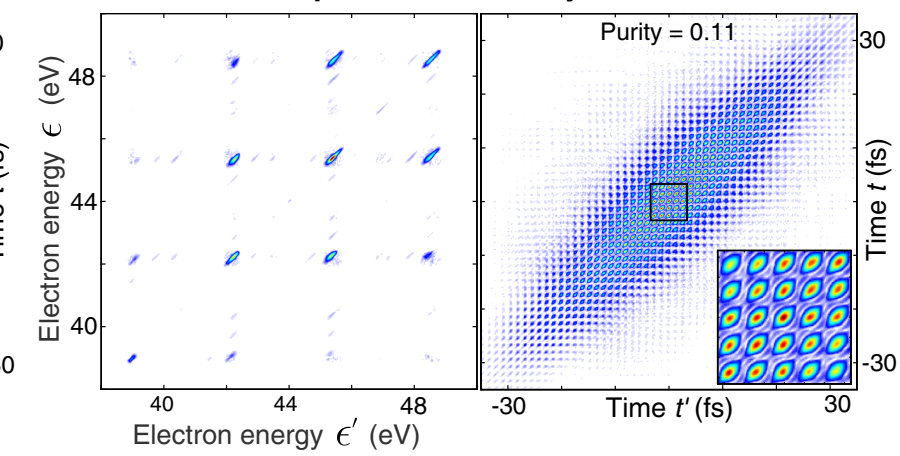

FIG. 8. For each decoherence model (a)-(e), the density matrix $\rho_{\text {model }}$ is fitted onto the experimental density matrix $\rho_{\text {exp }}$ reported in (f). In each case, the density matrix is depicted in the energy domain (left) and in the time domain (right); only the modulus is shown. 
attochirp fluctuations. They thus poorly describe the experimental mixture, which can also be seen from the shape and purity of the fitted density matrices in Figs. 8(a) and 8(b). More precisely, the elongated aspect of the attosecond peaks in $\rho_{\exp }\left(t, t^{\prime}\right)$ seems well reproduced but not the femtosecond structure. Indeed, the fitting algorithm assumes that the XUV partial coherence impacts only the broadband energy scale or, equivalently, the attosecond timescale. Therefore, fitting the density matrix with these two models implies that the femtosecond structure is not reproduced.

The error is decreased with the model accounting for the detector's response, and the purity of the modeled mixture goes down to 0.22 and comes closer to the experimental one; see Fig. 8(c). This result indicates that the spectrometer must be playing a more important role than XUV partial coherence in the final mixture. Nevertheless, this fit is not satisfactory, as high-order coherences in the energy domain are overestimated. Indeed, the present model considers that the detector's response influences only the narrow-band sub-eV aspect of the coherences. It is thus unable to reproduce the attosecond structure in the time domain.

\section{Models with two sources of decoherence}

The last two models combine XUV arrival-time fluctuations or attochirp variations with the detector's response. These more complex models can reproduce experimental data much more faithfully, and the obtained least-squares error is unsurprisingly much lower than before. Interestingly, the final error is twice smaller for the "XUV arrival time fluctuations + detector's response" model than with the "attochirp variations + detector's response" model. The latter model overestimates high-order coherences in the energy domain, whereas the first model attenuates them to reproduce the experimental results. These different behaviors can be understood from the simulations in Figs. 7(b) and 7(c), where it can be seen that arrival-time variations tend to make higher-order coherences disappear, when attochirp variations maintain the intensity of some of them.

[1] P. Paul et al., Observation of a Train of Attosecond Pulses from High Harmonic Generation, Science 292, 1689 (2001).

[2] M. Hentschel, R. Kienberger, C. Spielmann, G. A. Reider, N. Milosevic, T. Brabec, P. Corkum, U. Heinzmann, M. Drescher, and F. Krausz, Attosecond Metrology, Nature (London) 414, 509 (2001).

[3] J. Itatani, F. Quéré, G. L. Yudin, M. Yu. Ivanov, F. Krausz, and P. B. Corkum, Attosecond Streak Camera, Phys. Rev. Lett. 88, 173903 (2002).

[4] Y. Mairesse and F. Quéré, Frequency-Resolved Optical Gating for Complete Reconstruction of Attosecond Bursts, Phys. Rev. A 71, 011401 (2005).
[5] E. Goulielmakis et al. Single-Cycle Nonlinear Optics, Science 320, 1614 (2008).

[6] K. T. Kim, D. K. Ko, J. Park, V. Tosa, and C. H. Nam, Complete Temporal Reconstruction of Attosecond HighHarmonic Pulse Trains, New J. Phys. 12, 083019 (2010).

[7] M. Chini, S. Gilbertson, S. D. Khan, and Z. Chang, Characterizing Ultrabroadband Attosecond Lasers, Opt. Express 18, 13006 (2010).

[8] P. D. Keathley, S. Bhardwaj, J. Moses, G. Laurent, and F. X. Kärtner, Volkov Transform Generalized Projection Algorithm for Attosecond Pulse Characterization, New J. Phys. 18, 073009 (2016).

[9] X. Zhao, H. Wei, Y. Wu, and C. D. Lin, Phase-Retrieval Algorithm for the Characterization of Broadband Single Attosecond Pulses, Phys. Rev. A 95, 043407 (2017).

[10] F. Quéré, Y. Mairesse, and J. Itatani, Temporal Characterization of Attosecond XUV Fields, J. Mod. Opt. 52, 339 (2005).

[11] V. Yakovlev, J. Gagnon, N. Karpowicz, and F. Krausz, Attosecond Streaking Enables the Measurement of Quantum Phase, Phys. Rev. Lett. 105, 073001 (2010).

[12] S. Haessler, B. Fabre, J. Higuet, J. Caillat, T. Ruchon, P. Breger, B. Carré, E. Constant, A. Maquet, E. Mével, P. Salières, R. Taïeb, and Y. Mairesse, Phase-Resolved Attosecond near-Threshold Photoionization of Molecular Nitrogen, Phys. Rev. A 80, 011404(R) (2009).

[13] M. Schultze et al., Delay in Photoemission, Science 328 , 1658 (2010).

[14] G. Sansone et al., Electron Localization Following Attosecond Molecular Photoionization, Nature (London) 465, 763 (2010).

[15] M. Ivanov and O. Smirnova, How Accurate Is the Attosecond Streak Camera?, Phys. Rev. Lett. 107, 213605 (2011).

[16] F. Calegari et al., Ultrafast Electron Dynamics in Phenylalanine Initiated by Attosecond Pulses, Science 346, 336 (2014).

[17] R. Locher, L. Castiglioni, M. Lucchini, M. Greif, L. Gallmann, J. Osterwalder, M. Hengsberger, and U. Keller, Energy-dependent Photoemission Delays from Noble Metal Surfaces by Attosecond Interferometry, Optica 2, 405 (2015).

[18] Z. Tao, C. Chen, T. Szilvasi, M. Keller, M. Mavrikakis, H. Kapteyn, and M. Murnane, Direct Time-Domain Observation of attosecond Final-State Lifetimes in Photoemission from Solids, Science 353, 62 (2016).

[19] M. Isinger et al., Photoionization in the Time and Frequency Domain, Science 358, 893 (2017).

[20] I. Jordan and H. J. Wörner, Extracting Attosecond Delays from Spectrally Overlapping Interferograms, J. Opt. 20, 024013 (2018).

[21] L. Seiffert et al., Attosecond Chronoscopy of Electron Scattering in Dielectric Nanoparticles, Nat. Phys. 13, 766 (2017).

[22] A. L. Cavalieri et al., Attosecond Spectroscopy in Condensed Matter, Nature (London) 449, 1029 (2007).

[23] R. Pazourek, S. Nagele, and J. Burgdörfer, Attosecond Chronoscopy of Photoemission, Rev. Mod. Phys. 87, 765 (2015).

[24] S. Pabst, L. Greenman, P. J. Ho, D. A. Mazziotti, and R. Santra, Decoherence in Attosecond Photoionization, Phys. Rev. Lett. 106, 053003 (2011). 
[25] V. Gruson et al., Attosecond Dynamics through a Fano Resonance: Monitoring the Birth of a Photoelectron, Science 354, 734 (2016).

[26] O. Smirnova, V. S. Yakovlev, and Armin Scrinzi, Quantum Coherence in the Time-Resolved Auger Measurement, Phys. Rev. Lett. 91, 253001 (2003).

[27] N. Rohringer and R. Santra, Multichannel Coherence in Strong-Field Ionization, Phys. Rev. A 79, 053402 (2009).

[28] E. Goulielmakis et al., Real-Time Observation of Valence Electron Motion, Nature (London) 466, 739 (2010).

[29] S. Carlström, J. Mauritsson, K. J. Schafer, A. L'Huillier, and M. Gisselbrecht, Quantum Coherence in Photo-Ionisation with Tailored XUV Pulses, J. Phys. B 51, 015201 (2018).

[30] A. I. Kuleff and L. S. Cederbaum, Ultrafast CorrelationDriven Electron Dynamics, J. Phys. B 47, 124002 (2014).

[31] H. Timmers, Z. Li, N. Shivaram, R. Santra, O. Vendrell, and A. Sandhu, Coherent Electron Hole Dynamics near a Conical Intersection, Phys. Rev. Lett. 113, 113003 (2014).

[32] M. Vacher, M. J. Bearpark, M. A. Robb, and J. P. Malhado, Electron Dynamics upon Ionization of Polyatomic Molecules: Coupling to Quantum Nuclear Motion and Decoherence, Phys. Rev. Lett. 118, 083001 (2017).

[33] E. Perfetto, D. Sangalli, A. Marini, and G. Stefanucci, Ultrafast Charge Migration in XUV Photoexcited Phenylalanine: A First-Principles Study Based on Real-Time Nonequilibrium Greens Functions, J. Phys. Chem. Lett. 9, 1353 (2018).

[34] M. Ruberti, P. Decleva, and V. Averbukh, Full Ab Initio Many-Electron Simulation of Attosecond Molecular PumpProbe Spectroscopy, J. Chem. Theory Comput. 14, 4991 (2018).

[35] C. Bourassin-Bouchet and M. E. Couprie, Partially Coherent Ultrafast Spectrography, Nat. Commun. 6, 6465 (2015).

[36] C. Kurtsiefer, T. Pfau, and J. Mlynek, Measurement of the Wigner Function of an Ensemble of Helium Atoms, Nature (London) 386, 150 (1997).

[37] D. Leibfried, D. M. Meekhof, B. E. King, C. Monroe, W. M. Itano, and D. J. Wineland, Experimental Determination of the Motional Quantum State of a Trapped Atom, Phys. Rev. Lett. 77, 4281 (1996).

[38] D. T. Smithey, M. Beck, M. G. Raymer, and A. Faridani, Measurement of the Wigner Distribution and the Density Matrix of a Light Mode Using Optical Homodyne Tomography: Application to Squeezed States and the Vacuum, Phys. Rev. Lett. 70, 1244 (1993).

[39] G. Breitenbach, S. Schiller, and J. Mlynek, Measurement of the Quantum States of Squeezed Light, Nature (London) 387, 471 (1997).

[40] M. Swoboda, J. M. Dahlström, T. Ruchon, P. Johnsson, J. Mauritsson, A. L'Huillier, and K. J. Schafer, Intensity Dependence of Laser-Assisted Attosecond Photoionization Spectra, Laser Phys. 19, 1591 (2009).

[41] S. J. Weber et al., Flexible Attosecond Beamline for High Harmonic Spectroscopy and XUV/Near-IR Pump Probe Experiments Requiring Long Acquisition Times, Rev. Sci. Instrum. 86, 033108 (2015).
[42] See Supplemental Material at http://link.aps.org/ supplemental/10.1103/PhysRevX.10.031048 for a description of the experimental setup, of the Mixed FROG inversion algorithm, of the density matrix fitting algorithm, and for details on the simulation of the multichannel electron wave packet.

[43] P. H. Bucksbaum, R. R. Freeman, M. Bashkansky, and T. J. McIlrath, Role of the Ponderomotive Potential in AboveThreshold Ionization, J. Opt. Soc. Am. B 4, 760 (1987).

[44] J. M. Schins, P. Breger, P. Agostini, R. C. Constantinescu, H. G. Muller, G. Grillon, A. Antonetti, and A. Mysyrowicz, Observation of Laser-Assisted Auger Decay in Argon, Phys. Rev. Lett. 73, 2180 (1994).

[45] A. Ourjoumtsev, R. Tualle-Brouri, J. Laurat, and P. Grangier, Generating Optical Schrödinger Kittens for Quantum Information Processing, Science 312, 83 (2006).

[46] E. Bocquillon, V. Freulon, J.-M. Berroir, P. Degiovanni, B. Plaçais, A. Cavanna, Y. Jin, and G. Féve, Coherence and Indistinguishability of Single Electrons Emitted by Independent Sources, Science 339, 1054 (2013).

[47] Y. Mairesse et al., Attosecond Synchronization of HighHarmonic Soft X-rays, Science 302, 1540 (2003).

[48] K. Varjú et al., Frequency Chirp of Harmonic and Attosecond Pulses, J. Mod. Opt. 52, 379 (2005).

[49] K. Varjú, Y. Mairesse, P. Agostini, P. Breger, B. Carré, L. J. Frasinski, E. Gustafsson, P. Johnsson, J. Mauritsson, H. Merdji, P. Monchicourt, A. L'Huillier, and P. Salières, Reconstruction of Attosecond Pulse Trains Using an Adiabatic Phase Expansion, Phys. Rev. Lett. 95, 243901 (2005).

[50] Q. Zhang, K. Zhao, and Z. Chang, High Resolution Electron Spectrometers for Characterizing the Contrast of Isolated 25 as Pulses, J. Electron Spectrosc. Relat. Phenom. 195, 48 (2014).

[51] S. Deléglise, I. Dotsenko, C. Sayrin, J. Bernu, M. Brune, J.-M. Raimond, and S. Haroche, Reconstruction of NonClassical Cavity Field States with Snapshots of their Decoherence, Nature (London) 455, 510 (2008).

[52] K. Hornberger, S. Uttenthaler, B. Brezger, L. Hackermüller, M. Arndt, and A. Zeilinger, Collisional Decoherence Observed in Matter Wave Interferometry, Phys. Rev. Lett. 90, 160401 (2003).

[53] J. Feist, O. Zatsarinny, S. Nagele, R. Pazourek, J. Burgdrfer, X. Guan, K. Bartschat, and B. I. Schneider, Time Delays for Attosecond Streaking in Photoionization of Neon, Phys. Rev. A 89, 033417 (2014).

[54] A. Feist, K. E. Echternkamp, J. Schauss, S. V. Yalunin, S. Schäfer, and C. Ropers, Quantum Coherent Optical Phase Modulation in an Ultrafast Transmission Electron Microscope, Nature (London) 521, 200 (2015).

[55] M. Ossiander et al., Attosecond Correlation Dynamics, Nat. Phys. 13, 280 (2017).

[56] I. A. Walmsley and C. Dorrer, Characterization of Ultrashort Electromagnetic Pulses, Adv. Opt. Photonics 1, 308 (2009).

[57] NIST Electron Elasting-Scattering Cross-Section Database, https://srdata.nist.gov/SRD64/Elastic. 